\title{
Influence of Nonbonded Interactions on Molecular Geometry and Energy: Calculations for Hydrocarbons Based on Urey-Bradley Field*
}

\author{
E. Jean Jacob, H. Bradford Thompson, $\dagger$ and L. S. Barteld \\ Department of Chemistry, Universily of Michigan, Ann Arbor, Michigan
}

(Received 13 June 1967)

\begin{abstract}
A modified Urey-Bradley potential energy function comprised of quadratic terms for bond stretches, bond-angle bends, and torsional displacements together with analytical expressions for pairwise nonbonded interactions was chosen to represent the force field for hydrocarbon molecules. Quadratic constants were taken from the spectroscopic U-B analyses of Schachtschneider and Snyder [Spectrochim. Acta 19, 117 (1963)], while the nonbonded functions adopted were those proposed by Bartell [J. Chem. Phys. 32, 827 (1960) ]. Reference bond angles for the quadratic terms were taken to be $109.5^{\circ}$ or $120^{\circ}$ for tetrahedral or trigonal coordination, respectively. Reference single-bond lengths and the torsional constant were adjusted to fit the experimental data for $\mathrm{CH}_{4}$ and $\mathrm{C}_{2} \mathrm{H}_{6}$. Double bonds and ring bonds in cyclopropyl compounds were considered to be rigid. The above selections served to establish a universal model force field for hydrocarbons with no remaining adjustable parameters. The potential energy functions for a variety of saturated hydrocarbons and several olefins and cyclopropyl derivatives were minimized with respect to independent structure parameters (i.e., bond stretches, bends, and internal rotations). Even though all $\mathrm{C}-\mathrm{H}$ (and $\mathrm{C}-\mathrm{C}$ ) bonds were input to be identical to those in $\mathrm{CH}_{4}$ (and $\mathrm{C}_{2} \mathrm{H}_{6}$ ) except for nonbonded environment, the bond lengths and angles corresponding to the minimum potential energy exhibited an appreciable variation from molecule to molecule, as did also the strain energies of geometric and rotational isomers. Calculated trends in structures, isomerization energies, and barriers to rotation agreed quite well with experimentally observed trends, provided that experimental isomerization energies were corrected to $0^{\circ} \mathrm{K}$ and zero-point energies were taken into account. Some novel features of the results and applications of the model for predicting deformations in strained systems are discussed. The present study differs from previous work in the area of "molecular mechanics" in the use of a more general force field, in allowing the strained molecules to relax in all degrees of freedom (except for unsaturated groups and cyclopropyl rings), in the selection of molecular systems, and in a detailed comparison with experiment.
\end{abstract}

\section{INTRODUCTION}

Several decades have passed since chemists first addressed themselves seriously to the problem of steric effects in chemical systems. Kistiakowsky's early speculations on the structural and energetic effects of steric interactions pointed out the need for refined experimental data in a number of areas before quantitative treatments of the problem would be feasible. ${ }^{1}$ This need has now been met to a large extent in the areas of structure determination, thermodynamic measurement, and conformational analysis. On the other hand, attempts to interpret and interrelate the various experimental results in a self-consistent way have only recently been initiated. The impediment to progress was not so much the difficulty in formulating model force fields-the popular models of today are modest modifications of models formulated 20 years ago ${ }^{2}$-but rather in the means for handling the tedious calculations involved.

The rapidly growing field of "molecular mechanics" has been aided tremendously by the increased availability and capacity of high-speed digital computers. A number of refinements in the application of the basic technique have been developed as a result of this aug-

* This research was supported by a grant from the National Science Foundation.

$\dagger$ Present address: Department of Chemistry, University of Toledo, Ohio.

I J. B. Conn, G, B. Kistiakowsky, and E. A. Smith, J. Am. Chem. Soc. 61, 1868 (1939).

2 T. L. Hill, J. Chem. Phys. 14, 465 (1946); I. Dostrovsky, E. D. Hughes, and C. K. Ingold, J. Chem. Soc. 1946, 173; J. H. Westheimer and J. E. Mayer, J. Chem. Phys. 14, 733 (1946). mented computational ability. ${ }^{3-9}$ One of the most important of these has been the inclusion of structural deformations to relieve local steric strain in molecules. While recent papers ${ }^{6-9}$ have considered the effect on conformational energy differences of the adjustment of structural parameters of molecules to changes in the environment of nonbonded interactions, to date the task of minimizing the conformational energy with respect to all internal coordinates of complex molecules has usually been considered either too formidable or too unrewarding with the result that minimization has been restricted to selected rotational and angular deformations. This simplified approach undoubtedly yields satisfactory results when the source of the deformations is a single strong interaction, as in gauche conformations of the $n$-alkanes, or even a well-localized set of strong interactions.

In addition to deformations which are universally acknowledged to be steric, there are variations in bond

${ }^{3}$ The list of calculations performed in just the last five years is far too long to be presented here. References $(4)-(5)$ are representative of recent work done on bond energies, while Refs. (6) - (9) give a cross section of recent calculations on conformational energy differences. Useful information concerning force constants, barriers to internal rotation, and nonbonded potential functions currently in use can also be found in the indicated references.

${ }^{4}$ M. Cignitti and T. L. Allen, J. Chem. Phys. 43, 4472 (1965).

${ }^{B}$ A. J. Kalb, A. L. H. Chung, and T. L. Allen, J. Am. Chem. Soc. 88, 2938 (1966).

${ }^{6} \mathrm{~J}$. B. Hendrickson, J. Am. Chem. Soc. 86, 4854 (1964).

7 K. B. Wiberg, J. Am. Chem. Soc. 87, 1070 (1965).

${ }^{8}$ R. A. Scott and H. A. Scheraga, J. Chem. Phys. 44, 3054 (1966).

P. E. McMahon and R. L. McCullough, J. Phys. Chem. 69, $1747(1965)$. 
lengths and bond angles with changes in environment which are often attributed to changes in hybridization or to hyperconjugative interactions. The observation has been made that, at least for hydrocarbons, many of these variations can be formally accounted for on the basis of nonbonded interactions. ${ }^{10,11}$ Crude calculations suggested that the order of magnitude of the steric effect was sufficient, but calculations in which all internal coordinates of the molecule were allowed to adjust properly were never made.

Wiberg's calculations on some cycloalkanes ${ }^{7}$ have allowed full freedom to the structural parameters, but the choice of reference configurations makes the results suitable only for comparisons of energies between rotational conformations of the same molecule. This approach does not elucidate the effect of nonbonded interactions on molecular structures in a manner which provides quantitative information which is transferable to other molecules, nor does it provide values which are suitable for comparison with experimental values. It was the purpose of the present work to test the ability of a simple mechanical model to reproduce the details of structures of a variety of hydrocarbons. In addition, a study was made of the assumption used in many calculations that the relaxation of bond lengths and bond angles contributes only slightly to conformational energy differences.

The treatment described below is not intended to be theoretically rigorous insofar as individual components of the force field are concerned. It is rigorous within the framework of its simplified force field, however, in its derivation of molecular configurations corresponding to potential energy minima. Because the original focus of these calculations was on structural parameters, the force constants and nonbonded potential functions were not chosen with a view to reproducing thermochemical trends. Fairly good agreement with experimental thermodynamic data was achieved nonetheless.

The choice of molecules for this investigation was governed by two considerations. One was the obvious necessity of treating nonpolar (or nearly nonpolar) molecules to avoid the additional complexities due to permanent electric dipoles and to electronegativity effects. The other was the accumulation of a number of accurate experimental structural determinations for gaseous hydrocarbons with which to compare the calculated results. A secondary consideration was the availability and apparent transferability of UreyBradley force constants for hydrocarbons.

Differences in operational definitions of internuclear distance parameters for spectroscopy and gas diffraction experiments have given rise to apparent inconsistencies between bond lengths determined by the two methods. ${ }^{12}$

${ }^{10}$ L. S. Bartell, J. Chem. Phys. 32, 827 (1960).

11 L. S. Bartell, Tetrahedron 17, 177 (1962).

${ }^{12}$ L. S. Bartell, E. A. Roth, C. D. Hollowell, K. Kuchitsu, and J. E. Young, Jr., J. Chem. Phys. 42, 2683 (1965).
At present, a greater body of consistent structural information for hydrocarbons is available from electron diffraction than from other sources. For this reason comparisons in this paper between calculated and experimental results were made with electron diffraction data wherever reliable structures were available.

\section{CALCULATIONS}

\section{A. Model Force Field}

The calculations presented below are based on a modified Urey-Bradley force field which differs from the conventional Urey-Bradley field in two respects. First, it includes all nonbonded interactions in a molecule instead of only the geminal or other close interactions. Second, it expresses nonbonded interactions in terms of flexible anharmonic functions of internuclear distance instead of linear and quadratic functions of internuclear distance displacements from equilibrium. The functional form adopted for the nonbonded potential energy expression was

$$
V_{n b}(r)=\sum_{i} a_{i} r^{b_{i}} \exp \left(-c_{i} r\right)
$$

in which $r$ represents the distance between the nonbonded atoms involved. The most common nonbonded functions, the Lennard-Jones (12-6) and the Buckingham (exp-6) functions, can be expressed in this form. One function, $V_{n b}$, was specified for each type of interaction, e.g., C $\cdots \mathrm{C}, \mathrm{C} \cdots \mathrm{H}$, etc.

According to the model force field described above, the total potential energy of a molecule is given by

$$
\left.V_{\text {total }}=\sum_{n} V_{n b^{n}}\left(r_{n}\right)+\frac{1}{2} \sum_{l} k_{l}\left(\phi_{l}-\phi_{l}\right)^{2}\right)^{2},
$$

in which $n$ indexes the nonbonded internuclear distances, $V_{n b}{ }^{n}$ denotes the $V_{n b}$ corresponding to the distance $r_{n}, \phi_{l}$ is the $l$ th internal coordinate (bond length, bond angle, or dihedral angle), and superscript 0 identifies the reference value of $\phi_{l}$. The selection of the $V_{n b}$ 's, the force constants, and the reference values of the internal coordinates is discussed below.

\section{B. Energy Minimization Process}

\section{The Computer Program}

For all but the simplest molecules, the minimization of $V_{\text {total }}$ of Eq. (2) with respect to a set of independent structural parameters requires the use of a high-speed electronic computer. The calculations reported below were performed with programs written in FORTRAN II. The present program can vary thirty independent parameters simultaneously, and can sum over 200 internuclear distances. For most of the molecules studied, these dimensions were sufficient to allow all internal coordinates to vary when the molecules were constrained to suitable symmetries. In general, the symmetry imposed on a molecule was the lowest one for 
which no more than 30 parameters were necessary to allow every internal coordinate to vary. In a few cases (some of the branched hexanes and heptanes) some constraints on bond lengths and bond angles not demanded by symmetry were imposed.

\section{Description of Molecular Geometry and Method of Defining Variables}

The molecular description input to the computer consists of a set of internal coordinates, $\left\{\xi_{i}\right\}$, which is sufficient to determine the relative spatial positions of atoms in the molecule. Some or all of these internal coordinates may be allowed to vary during a given calculation. In order to impose a desired symmetry on a molecule, a set of independent variables $\left\{\theta_{i}\right\}$ were so defined that each $\xi_{l}$ could be expressed as

$$
\xi_{l}=A_{l}+B_{l} \theta_{j}
$$

in which $A_{l}$ and $B_{l}$ are constants. It is the $A_{l}, B_{l}$, and $j$, the index of the appropriate independent parameter, which are supplied to the program to specify $\xi_{l}$. The judicious choice of $A_{l}$ 's and $B_{l}$ 's and the relation of more than one $\xi_{l}$ to the same $\theta_{j}$ generates the desired symmetry elements in the molecule. This can greatly reduce the number of variables required for the minimization process.

The set of internal coordinates, $\left\{\xi_{i}\right\}$, adopted to describe the geometry of the molecule includes all bond lengths, and some but not all bond angles and dihedral angles. No matter how the $\left\{\xi_{i}\right\}$ are chosen, for atoms of coordination number greater than three it is impossible to include all bond angles in the $\left\{\xi_{i}\right\}$ without introducing redundancies. Accordingly, there will usually be a set of bond angles, $\left\{\zeta_{i}\right\}$, which are functions of the $\left\{\xi_{i}\right\}$, but which are not contained explicitly in the input molecular description. Variations in the $\left\{\zeta_{i}\right\}$ will affect the total energy of the molecule, so that the sum over all internal coordinates in Eq. (2) must be a sum over both $\left\{\xi_{i}\right\}$ and $\left\{\zeta_{i}\right\}$. Furthermore, of the dihedral angles specified in $\left\{\xi_{i}\right\}$, at most one per $\mathrm{C}-\mathrm{C}$ bond can be associated with an internal rotation in the expression for $V_{\text {total }}$. (For the calculations discussed in this paper, the two planes used to define a particular dihedral angle are restricted to planes which are determined by adjacent bonds in the molecule and which have one bond in common.) The remaining dihedral angles, which are related to the $\left\{\zeta_{i}\right\}$, are given zero force constants. In this way, every internal coordinate, $\phi_{l}$, is counted exactly once in $V_{\text {total }}, E q$. (2). It can be seen from Eq. (5) of the following section that the treatment of the $\left\{\zeta_{i}\right\}$ in the energy minimization procedure is mathematically completely analogous to the treatment of the nonbonded distances $r_{n}$.

\section{Mathematical Formulation of the Minimization of the Potential Energy}

A Gauss-Newton method for minimizing the total potential energy of a molecule with respect to the set of independent variables, $\left\{\theta_{i}\right\}$, was used. The values of $\left\{\theta_{i}\right\}$ which correspond to the configuration of lowest energy (subject to the constraints imposed on the molecule and the assumptions implicit in this model) were found by an iterative process. In the expansion of the functions $V_{n b}$ of Eq. (1), the substitution

$$
\left(r_{n}-r_{n}{ }^{w}\right)=\sum_{i}\left(\partial r_{n} / \partial \theta_{i}\right)_{w}\left(\theta_{i}-\theta_{i}{ }^{w}\right)
$$

was made. Superscript $w$ denotes the working values of the parameter in the iterative process. Subscript $w$ indicates that the derivative is evaluated for the set of working values $\left\{\theta_{i}{ }^{w}\right\}$.

At a minimum in the potential energy the derivative of $V_{\text {total }}$ with respect to any independent variable must be zero. This condition generates a set of simultaneous equations, one for each independent variable, which are linear in the correction parameters $\left\{\left(\theta_{i}-\theta_{i}{ }^{*}\right)\right\}$. The general form of these equations is given by

$$
\begin{aligned}
\left(\frac{\partial V_{\text {total }}}{\partial \theta_{m}}\right)_{w}= & 0=k_{m} S_{m}\left(\theta_{m}-\theta_{m}^{w}\right)+k_{m} S_{m}\left(\theta_{m}^{w}-\theta_{m}^{0}\right) \\
& +\sum_{j} k_{j}\left(\zeta_{j}^{w}-\zeta_{j}^{0}\right)\left(\frac{\partial \zeta_{j}}{\partial \theta_{m}}\right)_{w} \\
& +\sum_{i} \sum_{j} k_{j}\left(\frac{\zeta_{j}}{\partial \theta_{i}}\right)_{w}\left(\frac{\partial \zeta_{j}}{\partial \theta_{m}}\right)_{w}\left(\theta_{i}-\theta_{i}^{w}\right) \\
& +\sum_{n}\left(\frac{\partial V_{n b^{n}}}{\partial r_{n}}\right)_{w}\left(\frac{\partial r_{n}}{\partial \theta_{m}}\right)_{w} \\
& +\sum_{i} \sum_{n}\left(\frac{\partial^{2} V_{n b^{n}}}{\partial r_{n}{ }^{2}}\right)_{w}\left(\frac{\partial r_{n}}{\partial \theta_{i}}\right)_{w}\left(\frac{\partial r_{n}}{\partial \theta_{m}}\right)_{w}\left(\theta_{i}-\theta_{i}^{w}\right)
\end{aligned}
$$

The sum over $\left\{\phi_{l}\right\}$ in Eq. (2) has been replaced in Eq. (5) by sums over $\left\{\xi_{i}\right\}$ and $\left\{\zeta_{i}\right\}$. However, the sum over $\left\{\xi_{i}\right\}$ can be reduced to a sum over the independent variables, $\left\{\theta_{i}\right\}$, with appropriate multiplicative factors, $S_{i}$, where $S_{i}$ is defined as

$$
S_{i}=\sum_{l}\left|B_{l}^{i}\right| \text {. }
$$

The symbol $S_{i}$ represents the multiplicity of $\theta_{i}$ in the molecular description. In Eq. (5), $i$ indexes independent variables, $j$ indexes dependent bond angles, and $n$ indexes internuclear distances.

Each solution vector for the set of Eq. (5) is added to the vector $\mathbf{Q}^{w}$ to obtain a new $\mathbf{\Theta}^{w}$. Then the minimization process is repeated until the individual corrections become arbitrarily small and the energy is no longer lowered by application of the solution vector to the $\mathbf{Q}^{n}$ vector.

\section{Evaluation of Matrix Elements}

While it is usually simpler to describe the geometry of a molecule in terms of internal coordinates rather 
that Cartesian coordinates, the calculation of internuclear distances is more tractable in a Cartesian coordinate system. A subroutine was used which transformed the input internal coordinates into Cartesian coordinates in a fixed coordinate system for the molecule. ${ }^{13}$ The calculations of the Cartesian coordinates are based on the well-known transformations between arbitrary Cartesian systems via Eulerian angles, which can be related directly to selected bond angles and dihedral angles in the molecule. A sequence of coordinate transformations is employed to transform each atom in the molecule back to the reference coordinate system. (The origin of the reference system is usually chosen to be on some atom in the molecule or on a symmetry element.) The $r_{n}$ 's are evaluated directly from the Cartesian coordinates of the two atoms which define each distance. The derivatives, $\left(\partial r_{n} / \partial \theta_{i}\right)$, were evaluated exactly from the derivatives of the Cartesian coordinates with respect to $\theta_{i}$. The latter were calculated by substituting the derivative matrix of the appropriate transformation matrix into the sequence of matrices. In principle, the higher-order derivatives of $r_{n}$ could be obtained in an analogous way. The truncation of the series expansion of $r_{n}$ at the first-order terms was dictated by limited storage in the computers and by the (assumed) unfavorable return in improved convergence to a minimum for the time invested in computing all the $\left(\partial^{2} \boldsymbol{r}_{n} / \partial \theta_{i} \partial \theta_{j}\right)$ 's for each $\boldsymbol{r}_{n}$ rather than by an inability to evaluate these terms. The $\zeta_{j}$ 's are evaluated from the Cartesian coordinates of the atoms which define the $\zeta_{j}$ 's;

$$
\left(\partial \zeta_{j} / \partial \theta_{i}\right)=\left(\partial \zeta_{j} / \partial r_{j}\right)\left(\partial r_{j} / \partial \theta_{i}\right)
$$

is used to calculate the $\left(\partial \zeta_{j} / \partial \theta_{i}\right)$ terms in Eq. (5). In Eq. (7) $r_{j}$ denotes the nonbonded distance defined by the terminal atoms which specify $\zeta_{j}$. The $\left(\partial \zeta_{j} / \partial r_{j}\right)$ terms in Eq. (7) are calculated from the differentiation of the law of cosines for the angle $\zeta_{j}$. The derivatives $\left(\partial r_{j} / \partial \theta_{i}\right)$ are calculated in the manner described above for nonbonded distances in the molecule. The $\left(\partial V_{n b} / \partial r_{n}\right)$ 's and $\left(\partial^{2} V_{n b} / \partial r_{n}^{2}\right)$ 's are evaluated analytically.

\section{Reliability of the Numerical Solution}

Tests of the program indicated that convergence of the parameters was reached in from four to eight cycles in typical cases. Even for quite poor initial guesses of the values of the parameters the calculations converged readily, and the converged structure for a given molecule was essentially independent of the initial values chosen for the parameters. Representative uncertainties in converged values for individual parameters are $\pm 0.0001 \AA$ for bond lengths, $\pm 0.001^{\circ}$ for bond angles, and $\pm 0.005^{\circ}$ for dihedral angles defining internal rotation. The uncertainty increases slightly as the size of the molecule and consequently the number

\footnotetext{
${ }^{13}$ The geometrical calculations are described in detail in $\mathrm{H} . \mathrm{B}$. Thompson, J. Chem. Phys. 47, 3407 (1967).
}

of degrees of freedom increases. The final energy was reproducible to $\pm 0.01 \mathrm{cal} / \mathrm{mole}$ in nearly all cases. There was no indication that the minima found were not real and unique. Roundoff errors in the calculation of the total energy appeared to be a large factor limiting the reproducibility of the results.

\section{Selection of Parameters for the Force Field}

The molecular structure and potential energy corresponding to a solution of the minimization problem of Eq. (5) will depend, of course, upon the parameters chosen to specify the potential function of Eq. (2). In this preliminary study the trends from molecule are of more importance than the exact values for a given molecule. Presumably these trends will be less sensitive to imperfections in the model force field than will the absolute values. Therefore, in the selection of input parameters and functions an attempt was made (1) to use the most appropriate published data wherever possible (e.g., force constants and experimental structures from which are obtained reference bond lengths), (2) to adopt the simplest reference values for bond angles and torsional angles, and (3) to employ a moderate, mutually consistent set of nonbonded potential functions, avoiding the extremes sometimes invoked in this poorly understood field of research. The input parameters initially chosen on this basis were employed in all calculations described in the following with no further adjustments in the course of research to improve agreement with experiment.

\section{Nonbonded Potential Functions}

The potential functions for nonbonded interactions chosen for this study were those published several years ago ${ }^{10}$ in an examination of the effect of coordination number on bond length. They are given in kilocalories per mole by

$$
\begin{aligned}
& V_{\mathrm{CC}}(r)=2.993 \times 10^{5} r^{-12}-3.252 \times 10^{2} r^{-6} \\
& V_{\mathrm{CH}}(r)=4.471 \times 10^{4} \exp (-2.04 r) r^{-6}-1.249 \times 10^{2} r^{-6}
\end{aligned}
$$

$$
V_{\mathrm{HH}}(r)=6.591 \times 10^{3} \exp (-4.08 r)-49.2 r^{-6},
$$

where $r$ is in Angstrom units. These functions were constructed, as noted in the original article, from a somewhat arbitrary combination of empirical and semiempirical data. They were not devised to reproduce the spectroscopic Urey-Bradley nonbonded force constants, $F_{i j}$, but were found, nevertheless, to give second derivatives for $\mathrm{C} \cdots \mathrm{C}$ and $\mathrm{C} \cdots \mathrm{H}$ interactions of roughly the observed magnitude. On the other hand, the values of $F_{\mathrm{HH}}$ yielded by conventional UreyBradley analyses are much smaller than those of $F_{\mathrm{CH}}$ and $F_{\mathrm{CC}}$ and much smaller than the second derivative of Eq. (10) at the mean $\mathrm{H} \cdots \mathrm{H}$ distance. This discrepancy seems as likely to be an artifact of the conventional Urey-Bradley analysis as it is a diagnosis of 
TABLE I. Force constants."

\begin{tabular}{cc} 
Coordinate & $\begin{array}{c}\text { Experimental } \\
\text { value }^{\mathrm{b}}\end{array}$ \\
\hline Bond length & 2.2 \\
$K_{\mathrm{CC}}$ & 4.1 \\
$K_{\mathrm{CH}}$ & \\
Bond angle & 0.687 \\
$H_{\mathrm{CCC}}$ & 0.320 \\
$H_{\mathrm{HCC}}$ & 0.520 \\
$H_{\mathrm{BCH}}$ & \\
Rotation about $R_{\mathrm{CC}}$ & $0.0853^{\circ}$ \\
$H_{r}$ & \\
\hline
\end{tabular}

a Constants taken from Schachtschneider and Snyder, Ref. 15, as explained in text, unless otherwise indicated.

b $K^{\prime} s$ in units of millidynes per angstrom. $H^{\prime} s$ in units of millidyne. angstroms per radian.:

' See text.

errors in Eq. (10). In evidence of this, a modified UreyBradley analysis of several hydrides ${ }^{14}$ gave nonbonded force constants which were entirely compatible with Eq. (10) when anharmonicity and interactions neglected in conventional analyses were taken into account. The deficiencies of conventional analyses are probably less severe for $\mathrm{C} \cdots \mathrm{H}$ and $\mathrm{C} \cdots \mathrm{C}$ interactions than for $\mathrm{H} \cdot \mathrm{HH}$ but are not negligible. Therefore the fact that Eqs. (8)-(10) do not reproduce closely the UreyBradley constants reported for hydrocarbons in the comprehensive study of Schachtschneider and Synder ${ }^{15}$ is not regarded as definitive evidence against the potentials of Eqs. (8)-(10). By the same token, no claim is made for the essential correctness of Eqs. (8)-(10). These functions have been compared by several authors ${ }^{4,16}$ with various other proposed functions and found to be of intermediate magnitude and hardness. Therefore it was felt that they might give a plausible starting point for assessments of nonbonded effects in hydrocarbons.

\section{Force Constants}

The force constants for bond stretches and angle deformations were taken from values reported by Schachtschneider and Snyder ${ }^{15}$ for saturated hydrocarbons in Urey-Bradley force fields. Because the force field defined for the calculations discussed below specifically includes all pairwise nonbonded interactions, it would be inconsistent to use valence force

${ }^{14}$ L. S. Bartell and K. Kuchitsu, J. Chem. Phys. 37, 691 (1962).

I6 J. H. Schachtschneider and R. G. Snyder, Spectrochim. Acta 19,117 (1963).

${ }_{16}^{16}$ J. B. Hendrickson, J. Am. Chem. Soc. 83, 4537 (1961). Note that Hendrickson uses valence force field constants in his model field and superimposes nonbonded interactions. Since this approach effectively counts the stronger nonbonded interactions twice, it is not surprising that the scheme of Hendrickson rejected strong nonbonded potential functions. A Urey-Bradley field rather than a valence feld must be used if nonbonded interactions are invoked between closest pairs of nonbonded atoms. constants. In order to simplify the model and reduce the number of force constants needed for the calculation of hydrocarbon structures, a somewhat arbitrary assumption about the nature of the molecular force field was made. It was assumed that a particular kind of internal coordinate, e.g., $\mathrm{C}-\mathrm{C}$ bond, $\mathrm{C}-\mathrm{H}$ bond, $\mathrm{CCC}$ angle, etc., was identical in all molecules except for its nonbonded environment. This implies that the force constant for a given type of coordinate is transferrable not only from methyl group to methyl group, say, but from methyl group to methylene group as well. Table I gives the actual constants used in the calculations reported below. In some cases they are averages of several values reported according to the above assumption. It should be said in defense of this assumption that Urey-Bradley force constants are reasonably transferrable from molecule to molecule, and that the site-to-site variations are small.

A further arbitrary simplification was made. As discussed in the preceding section, the nonbonded potential functions of Eqs. (8)-(10) are not completely consistent with the empirical force constants of Schachtschneider and Snyder. ${ }^{15}$ Perhaps the least arbitrary way to minimize the discrepancy, yet make use of functions such as Eqs. (8)-(10), would be to rederive a set of stretch and bend constants which, together with the nonbonded potentials adopted, would best fit the molecular spectra. At this preliminary stage of exploration, however, the modest increase in accuracy would not seem to warrant the additional labor. Therefore, no attempt was made to modify force constants to achieve complete compatibility with frequencies of molecular vibrations.

\section{Torsional Potential Function}

The force constant for rotation about a $\mathrm{C}-\mathrm{C}$ single bond was evaluated from the experimental barrier height in ethane. The energy for rotation about a bond can be expressed as a Fourier cosine series, given by Eq. (11),

$$
V_{\text {rot }}=\frac{1}{2} \sum_{m} V_{m}\left\{1-\cos \left[m\left(\beta-\beta^{0}\right)\right]\right\},
$$

where $\beta^{0}$ is the dihedral angle of the minimum energy configuration. Usually only a single variable is used to fit the experimental data, in which case $V_{n}$ represents the height of a simple $n$-fold barrier. In the present calculations, which deal with small values of $\left(\beta-\beta^{0}\right)$, $V_{\text {rot }}$ was simplified to

$$
V_{\text {rot }}=\frac{1}{2} k_{\text {rot }}\left(\beta-\beta^{0}\right)^{2},
$$

where $k_{\text {rot }}=\frac{1}{2} n^{2} V_{n}$. For the three-fold barrier in ethane, with $V_{3}$ taken as $3 \mathrm{kcal} / \mathrm{mole}, k_{\text {rot }}$ has the value of $13.5 \mathrm{kcal} \mathrm{mole}{ }^{-1} \cdot \mathrm{rad}^{-2}$.

It was assumed, for sake of argument, that in each molecule the barrier consisted of a part which was characteristic of the $\mathrm{C}-\mathrm{C}$ bond itself, independent of the bond environment, and of a steric part. This as- 
sumption was not intended to imply anything about the physical origin of the barrier to rotation. Rather it was made since it seemed the least arbitrary scheme for reproducing the general magnitude of barriers while taking into account the demonstrably steric interactions which occur in some of the molecules studied (c.f. hexamethylethane). The part of the barrier taken to be characteristic of a C-C single bond, and thus considered to be constant for all bonds between two fourcoordinate carbons in hydrocarbon molecules, was evaluated by subtracting from the experimental $V_{3}$ for ethane the calculated $270-\mathrm{cal} / \mathrm{mole}$ energy difference between eclipsed and staggered ethane. ${ }^{17}$ The corrected $k_{\text {rot }}$ of $12.28 \mathrm{kcal} \mathrm{mole}{ }^{-1} \cdot \mathrm{rad}^{-2}$ is only about $10 \%$ smaller than that calculated directly from $V_{3}$. This reduced constant was used in all calculations with saturated hydrocarbons. Since threefold symmetry about $\mathrm{C}-\mathrm{C}$ bonds is not realized in any of the alkanes (except ethane, neopentane, and fully methylated derivatives of neopentane), the choice of the dihedral angle for defining the amount of rotation about a $\mathrm{C}-\mathrm{C}$ bond is somewhat arbitrary. However, the different choices should affect the position of the energy minimum and the values of $V_{\text {total }}$ at the minimum only slightly. The reference configurations adopted for all the alkanes were taken to be the normal staggered configurations, assuming that $120^{\circ}$ dihedral angles would exist at each carbon atom if there were no nonbonded interactions.

\section{Reference Values of Bond Angles and Bond Lengths}

In the alkane molecules studied, the bond angles $\alpha$ were all assumed to be $\alpha^{0}=109.47 \ldots{ }^{\circ}$, the tetrahedral angle, in the absence of nonbonded forces. Both $120^{\circ}$ and the tetrahedral angle were tried as reference values for angles about carbon atoms in the double bond of the olefins studied. The angles about fourcoordinate carbons in the olefins were referenced at the tetrahedral angle. In cyclopropane derivatives, the angles between adjacent exterior bonds were referenced both at the tetrahedral angle and at $120^{\circ}$. A choice of a reference value for the angles about carbon atoms in the cyclopropyl ring is even more arbitrary than in acyclic alkanes and olefins in view of the severe deformation of the ring CCC angle. The reference value, $R_{\mathrm{CH}^{0}}{ }^{0}$, that was adopted for all $\mathrm{C}-\mathrm{H}$ bonds was obtained by finding the value of $R_{\mathrm{CH}^{0}}{ }^{0}$ which reproduced the experimental $R_{\mathrm{CH}}$ in methane upon convergence to an energy minimum. An $R_{\mathrm{CH}^{0}}$ of $1.056 \AA$ gives an $R_{\mathrm{CH}}$ of $1.107 \AA$, in agreement with the experimental value. ${ }^{18}$ The reference value for a $\mathrm{C}-\mathrm{C}$ single bond was found by calculating the structure of ethane using $\alpha^{0} \mathrm{HCC}=$ $109.47^{\circ}$ together with the $R_{\mathrm{CH}}{ }^{0}$ from methane, and ad-

\footnotetext{
${ }^{17}$ Note that the energy difference referred to was calculated for the two configurations at fixed rotational angles. It is independent of any assumed barrier height.

${ }^{18}$ L. S. Bartell, K. Kuchitsu, and R. J. de Neui, J. Chem. Phys. 33, 1254 (1960).
}

justing the $R_{\mathrm{CC}}{ }^{0}$ until a good representation of experimental data was obtained. Since the value of $R_{\mathrm{CC}}{ }^{0}$ in ethane affects the calculated values of the HCC angle and $R_{\mathrm{CH}}$ as well as $R_{\mathrm{CC}}$, the $R_{\mathrm{CC}}{ }^{0}$ selected was a compromise to obtain the best over-all fit of the three structural parameters in ethane. An $R_{\mathrm{CC}}{ }^{0}$ value of $1.24 \AA$ gave $R_{\mathrm{CC}}=1.540 \AA, R_{\mathrm{CH}}=1.114 \AA$, and $\alpha_{\mathrm{HOC}}=111.4 .^{\circ}$ These compare with experimental values of $R_{\mathrm{CC}}=$ $1.534 \AA, R_{\mathrm{CH}}=1.112 \AA$, and $\alpha_{\mathrm{HCC}}=111.0^{\circ} .^{19}$ The same $R_{\mathrm{CH}^{0}}{ }^{0}$ and $R_{\mathrm{CC}}{ }^{0}$ were used throughout the remainder of the calculations. Because of the compromise accepted above it is not unexpected to find that calculated $\mathrm{C}-\mathrm{C}$ bond lengths tend to be slightly longer than observed lengths in the remainder of the molecules studied. Calculations on olefin (and cyclopropyl) systems were made on the basis of rigid double bonds (and ring bonds) to avoid introducing additional adjustable parameters.

\section{COMPARISON BETWEEN CALCULATED AND EXPERIMENTAL RESULTS}

\section{A. Structural Trends}

Although a number of calculations of the effects of nonbonded interactions on molecular structures have been made prior to this work, the majority of them did not allow all independent structural parameters to vary simultaneously in the course of the calculation. Wiberg's studies of several cycloalkanes ${ }^{7}$ are notable exceptions. In Wiberg's work the Cartesian coordinates of the atoms were varied using a method of steepest descent in order to find the energy minima of the molecules. Apart from some differences in choices of nonbonded potential functions and effective force constants the major differences between Wiberg's work and the results reported here are the use of a valence force field (neglecting geminal repulsions) instead of a Urey-Bradley field, Cartesian coordinates instead of internal coordinates, and the emphasis in Wiberg's work on the calculation of energy differences between rotational conformers in contrast to our emphasis on the calculation of detailed molecular structures. Wiberg selected as reference point values for his $\mathrm{C}-\mathrm{C}$ and $\mathrm{C}-\mathrm{H}$ bonds the approximate equilibrium values found in saturated hydrocarbons, e.g., 1.54 and $1.09 \AA$, respectively. His interest was in calculating the effect of deformations from "normal," unstrained configurations on preferred conformations rather than on the prediction of actual structural parameters. Hendrickson also included strain energy terms in his calculation of preferred conformations of cycloalkanes, ${ }^{6,16,20}$ though his minimization involved only dihedral angles and bond angles at sites of severe steric strain. The minimization was effected by a systematic search over combinations of the various parameters. Other calcula-

${ }^{19}$ L. S. Bartell and H. K. Higginbotham, J. Chem. Phys. 42, 851 (1965).

${ }_{20}$ J. B. Hendrickson, J. Am. Chem. Soc. 84, 3355 (1962). 
TABLE II. Calculated structures of selected molecules.^

\begin{tabular}{|c|c|c|c|c|c|}
\hline \multicolumn{3}{|l|}{ Diamond lattice ${ }^{b}$} & \multicolumn{3}{|l|}{ Neopentane, $T_{d}$} \\
\hline $\mathrm{CC}$ bond & & $1.5448 \AA$ & $\mathrm{CC}$ bond & (4) & $1.5464 \AA$ \\
\hline & & & $\mathrm{CH}$ bond & (12) & $1.1138 \AA$ \\
\hline Propane, $C_{2 v}$ & & & $\Varangle \mathrm{HCC}$ & (12) & $111.61^{\circ}$ \\
\hline $\mathrm{CC}$ bond & (2) & $1.5423 \AA$ & & & \\
\hline $\mathrm{CH}$ bond, $\mathrm{CH}_{2}$ & (2) & $1.1223 \AA$ & Hexamethylethane, $D_{3} \mathrm{~d}$ & & \\
\hline $\mathrm{CH}$ bond, $\mathrm{CH}_{3}$, in plane & (2) & $1.1142 \AA$ & $\mathrm{CC}$ bond, central & (1) & $1.5664 \AA$ \\
\hline $\mathrm{CH}$ bond, $\mathrm{CH}_{3}$, out of plane & (4) & $1.1139 \AA$ & $\mathrm{CC}$ bond, terminal & (6) & $1.5523 \AA$ \\
\hline$\Varangle \mathrm{CCC}$ & (1) & $111.10^{\circ}$ & CH bond, "gauche," close & (6) & $1.1105 \AA$ \\
\hline$\Varangle \mathrm{HCC}, \mathrm{CH}_{2}$ & (4) & $109.94^{\circ}$ & $\mathrm{CH}$ bond, "gauche," far & (6) & $1.1126 \AA$ \\
\hline$\Varangle \mathrm{HCC}, \mathrm{CH}_{3}$, in plane & (2) & $111.27^{\circ}$ & CH bond, "trans" & (6) & $1.1156 \AA$ \\
\hline$\Varangle \mathrm{HCC}, \mathrm{CH}_{3}$, out of plane & (4) & $111.55^{\circ}$ & $\Varangle \mathrm{CH}_{3}-\mathrm{C}-\mathrm{C}$ & (6) & $111.09^{\circ}$ \\
\hline$\Varangle \mathrm{HCH}, \mathrm{CH}_{3}$, i.p.-o.p. & (4) & $107.36^{\circ}$ & $\Varangle \mathrm{HCC}$, "gauche," close & (6) & $112.66^{\circ}$ \\
\hline & & & $\Varangle \mathrm{HCC}$, “gauche,” far & (6) & $112.10^{\circ}$ \\
\hline Isobutane, $C_{3}$ & & & $\Varangle \mathrm{HCC}$, "trans" & (6) & $111.04^{\circ}$ \\
\hline $\mathrm{CC}$ bond & (3) & $1.5438 \AA$ & $\Varangle \mathrm{HCH}$, "gauche"-“gauche" & (6) & $107.62^{\circ}$ \\
\hline $\mathrm{CH}$ bond, tertiary $\mathbf{H}$ & (1) & $1.1321 \AA$ & $\Varangle \mathrm{HCH}$, "trans"-"gauche," close & $(6)$ & $106.95^{\circ}$ \\
\hline $\mathrm{CH}$ bond, $\mathrm{CH}_{3}$, "equatorial" & (6) & $1.1140 \AA$ & Rotation of $\mathrm{CH}_{3}$ 's & $(6)$ & $5.58^{\circ}$ \\
\hline $\mathrm{CH}$ bond, $\mathrm{CH}_{3}$, "axial" & (3) & $1.1138 \AA$ & Rotation about central $\mathrm{CC}$ bond & (1) & $12.64^{\circ}$ \\
\hline$\Varangle \mathrm{CCC}$ & (3) & $110.41^{\circ}$ & & & \\
\hline$\Varangle \mathrm{HCC}, \mathrm{CH}_{3}$, "equatorial" & (6) & $111.45^{\circ}$ & 1,1-Dimethylcyclopropane, $C_{2 v}$ & & \\
\hline$\Varangle \mathrm{HCC}, \mathrm{CH}_{z}$, "axial" & (3) & $111.69^{\circ}$ & $\mathrm{CC}$ bond, ring (not varied) & (3) & $1.5110 \AA$ \\
\hline$\Varangle \mathrm{HCH}$, "axial"_“equatorial" & (6) & $107.40^{\circ}$ & $\mathrm{CC}$ bond, $\mathrm{CH}_{3}-\mathrm{C}_{\mathrm{ring}}$ & (2) & $1.5185 \AA$ \\
\hline & & & $\mathrm{CH}$ bond, ring $\mathrm{H}$ & (4) & $1.1072 \AA$ \\
\hline Isobutylene, $C_{2 v}$ & & & $\mathrm{CH}$ bond, $\mathrm{CH}_{3}$, over the ring & (2) & $1.1156 \AA$ \\
\hline $\mathrm{C}=\mathrm{C}$ bond (not varied) & (1) & $1.337 \AA$ & $\mathrm{CH}$ bond, $\mathrm{CH}_{3}$ & (4) & $1.1161 \AA$ \\
\hline $\mathrm{C}-\mathrm{C}$ bond & (2) & $1.5127 \AA$ & $\Varangle \mathrm{CH}_{3}-\mathrm{C}-\mathrm{CH}_{3} \mathrm{e}$ & (1) & $116.53^{\circ}$ \\
\hline $\mathrm{CH}$ bond, ethylenic & (2) & $1.1053 \AA$ & $\Varangle \mathrm{CH}_{3}-\mathrm{C}_{\text {ring }}-\mathrm{C}_{\text {ring }}$ & (4) & $117.10^{\circ}$ \\
\hline $\mathrm{CH}$ bond, $\mathrm{CH}_{3}$, cis to $\mathrm{C}=\mathrm{C}$ & (2) & $1.1160 \AA$ & $\Varangle \mathrm{H}-(\mathrm{CH})-\left(\mathrm{CH}_{2}\right)$ & (2) & $118.59^{\circ}$ \\
\hline $\mathrm{CH}$ bond, $\mathrm{CH}_{3}$, out of plane & (4) & $1.1167 \AA$ & $\Varangle \mathrm{H}-(\mathrm{CH})-\mathrm{C}\left(\mathrm{CH}_{3}\right)_{2}$ & (2) & $119.06^{\circ}$ \\
\hline $\begin{array}{l}\Varangle \mathrm{CH}_{3}-\mathrm{C}=\mathrm{Cc} \\
\Varangle \mathrm{H}-\mathrm{C}=\mathrm{Cc}\end{array}$ & $\begin{array}{l}(2) \\
(2)\end{array}$ & $\begin{array}{l}121.44^{\circ} \\
122.84^{\circ}\end{array}$ & Implied $\Varangle H C H$, exterior to ring & (2) & $112.33^{\circ}$ \\
\hline $\begin{array}{l}\Varangle \mathrm{H}-\mathrm{C}=\mathrm{C} \\
\Varangle \mathrm{HCC}, \mathrm{CH}_{3}, \mathrm{H} \text { ais to } \mathrm{C}=\mathrm{C}\end{array}$ & $\begin{array}{l}(2) \\
(2)\end{array}$ & $112.62^{\circ}$ & $\Varangle \mathrm{HCC}, \mathrm{CH}_{3}$, over the ring & (2) & $112.60^{\circ}$ \\
\hline$\Varangle \mathrm{HCC}, \mathrm{CH}_{3}, \mathrm{H}$ out of plane & (4) & $111.57^{\circ}$ & $\Varangle \mathrm{HCC}, \mathrm{CH}_{3}$ & (4) & $111.48^{\circ}$ \\
\hline$\Varangle \mathrm{HCH}, \mathrm{CH}_{3}$, o.p.-o.p. & (2) & $107.08^{\circ}$ & $\Varangle \mathrm{HCH}, \mathrm{CH}_{3}$ & (2) & $107.14^{\circ}$ \\
\hline
\end{tabular}

a The numbers in parentheses indicate the multiplicities of the parameters. The symmetries given for the molecules are those of the converged parameters. In each molecule, rotations about $\mathrm{CC}$ single bonds were allowed, and lower symmetries were available to the molecules as a consequence.

${ }^{b}$ For diamond, the energy per bond in an infinite lattice was represented by Eq. (2) and minimized with respect to the $\mathrm{C}-\mathrm{C}$ bond length. The sum over all nonbonded distances for a given atom was approximated by a discreet sum over the six shortest nonbonded distance types plus an integral over the remainder of the lattice. The lower limit of integration was taken as the radius of a sphere which enclosed all the atoms in the discreet summation and preserved the density of the bulk crystal. At the converged value of $R_{\mathrm{CC}}$, the last distance in the summation was $4.6343 \AA$, implying a

lower limit of integration of $4.9032 \AA$. Eighty-seven atoms were enclosed in the volume over which the discrete summation was made. All the interactions represented by the integral were attractive, and the total effect of the integral was a decrease in the bond length of approximately $0.0015 \AA$.

c $120^{\circ}$ reference angles about three-coordinate carbon atoms were assumed.

d All rotations are in the same direction when viewed along the $\mathrm{CC}$ bonds toward the center of the molecule, from reference configurations having $60^{\circ}$ dihedral angles.

Tetrahedral reference value for the $\mathrm{CCC}$ and $\mathrm{HCH}$ angles exterior to the ring were assumed.

tions by Allen and co-workers, ${ }^{5}$ Scott and Scheraga, ${ }^{8}$ McMahon and McCullough, ${ }^{9}$ and others ${ }^{21}$ have similarly emphasized energetic results, either for the prediction of minimum energy conformations, or for the treatment of variations of experimental bond energies from constant values.

General agreement between experimental structures and the structures calculated with our model was obtained for a variety of small hydrocarbons. Table II lists the calculated parameters of selected molecules to indicate the characteristics of representative results. It appears that the model gives a not unreasonable es-

timate of the force field for the molecules for which structures were calculated. Of more significance than results for individual molecules is the result that the calculated structures also reproduce observed trends in changes in bond lengths and bond angles with changes in the nonbonded environment. These trends are discussed below.

\section{Bond Lengths}

In electron-diffraction studies of alkanes, determinations of the mean bonded $\mathrm{C}-\mathrm{C}$ and $\mathrm{C}-\mathrm{H}$ distances can be made with much greater accuracy then the differences between nonequivalent bonds. In unstrained sat- 
urated hydrocarbons the experimental evidence is that variations in the length of a $\mathrm{C}-\mathrm{C}$ bond with changes in nonbonded environment are too small to be resolved in individual molecules. The calculated bond lengths for the alkanes are in agreement with this result. In the normal alkanes, the largest calculated difference between $\mathrm{C}-\mathrm{C}$ bonds in the same molecule was $0.004 \AA$ (in gauche-trans $n$-pentane). Experimentally, appreciable differences in $\mathrm{C}-\mathrm{C}$ bond length appear only with changes in coordination number, or with some similarly large change in steric environment. Single $\mathrm{C}-\mathrm{C}$ bonds adjacent to double or triple bonds, or to a cyclopropyl ring, for example, are shortened. On the other hand, the central $\mathrm{C}-\mathrm{C}$ bond in hexamethylethane, which suffers from severe crowding of hydrogens across the central bond, is considerably lengthened. In Fig. 1 a plot of calculated $\mathrm{C}-\mathrm{C}$ bond lengths versus the corresponding experimental values shows a positive correlation between the calculated and experimental bond lengths for a wide range of molecules.

While the entire range of experimental values for $\mathrm{C}-\mathrm{H}$ bond lengths is only half the spread observed for $\mathrm{C}-\mathrm{C}$ bonds $(0.04 \AA$ vs $0.08 \AA)$, in some respects the $\mathrm{C}-\mathrm{H}$ bonds seem more sensitive to the nonbonded environment than do $\mathrm{C}-\mathrm{C}$ bonds. In the series ethane, propane, isobutane, neopentane, the $\mathrm{C}-\mathrm{C}$ bond length remains constant to within experimental error. However, in the analogous series for $\mathrm{C}-\mathrm{H}$ bonds, i.e., tertiary, secondary, primary hydrogens and methane hydrogens, the experimental values span nearly the entire range of $\mathrm{C}-\mathrm{H}$ bond lengths. In Fig. 2 the plot of calculated versus experimental $\mathbf{C}-\mathbf{H}$ bond lengths illustrates how well the calculated values fit observed trends in these parameters. The calculated $\mathrm{C}-\mathrm{H}$ bond lengths exhibit characteristic values for methane, primary, secondary, and tertiary hydrogens of 1.107, 1.114, 1.122 , and $1.134 \AA$, respectively. The variation within

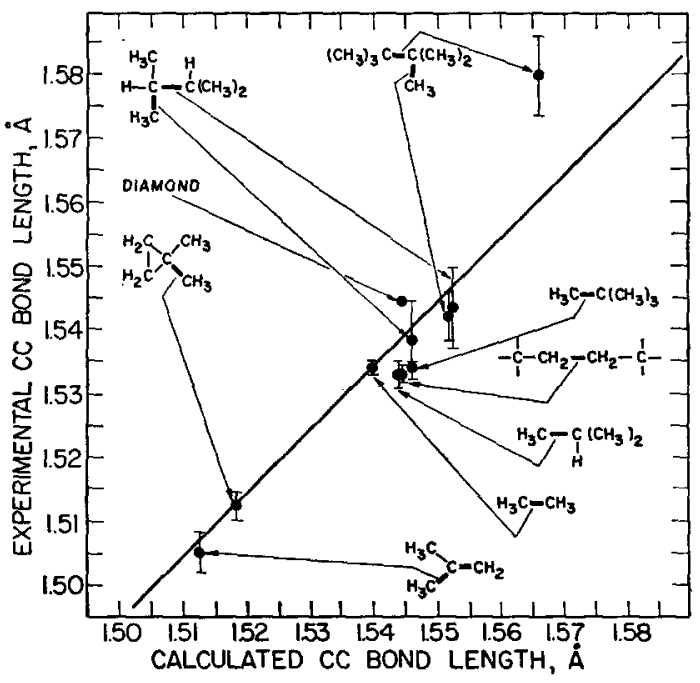

FIG. 1. Comparison of calculated and experimental $\mathrm{CC}$ bond lengths in hydrocarbons.

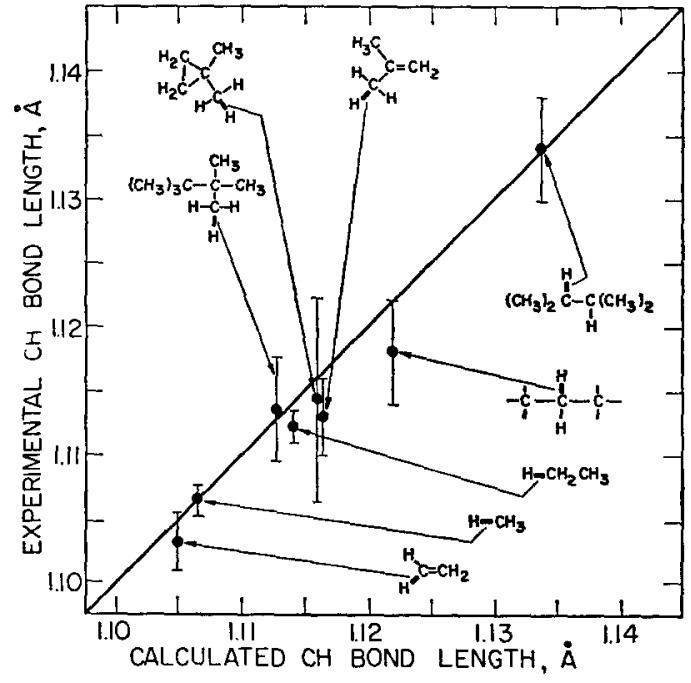

FIG. 2. Comparison of calculated and experimental $\mathrm{CH}$ bond lengths in hydrocarbons.

each grouping is less than the differences between the groups. The $\mathrm{C}-\mathrm{H}$ bonds in methyl groups of olefins and cyclopropane derivatives were within $0.003 \AA$ of the value for primary hydrogens in the alkanes, while the $1.107-\AA \mathrm{C}-\mathrm{H}$ bonds to secondary hydrogens on cyclopropyl rings were distinctly shorter. Since electron diffraction studies of hydrocarbons have been unable to resolve the different $\mathrm{C}-\mathrm{H}$ bond lengths in individual molecules, there is no direct experimental measure of the characteristic value for a secondary $\mathrm{C}-\mathrm{H}$ bond length available. A value for the secondary bond can be derived from the mean $\mathrm{C}-\mathrm{H}$ bond lengths observed ${ }^{22}$ for the series of molecules $\left(\mathrm{CH}_{3}\right)_{2}\left(\mathrm{CH}_{2}\right)_{n}$, however, if it is assumed that the primary bonds have the same length as in ethane. The resultant weighted average for the $R_{\mathrm{CH}}\left(2^{0} \mathrm{H}\right)$ 's for $n$-butane, $n$-pentane, $n$-hexane, and $n$-heptane is $1.118 \pm 0.004 \AA$. This experimental value is not significantly different from the calculated value of $1.122 \AA$, but is larger than the experimental value for primary hydrogens. The only experimental electrondiffraction value for a $\mathrm{C}-\mathrm{H}$ bond to a tertiary hydrogen is that for sym-tetramethylethane ${ }^{23}$ (2,3-dimethylbutane, abbreviated as TME in the following). Again, the $R_{\mathrm{CH}}\left(3^{\circ} \mathrm{H}\right)$ for TME is a derived quantity. A value of $R_{\mathrm{CH}}\left(3^{0}\right)=1.134 \pm 0.02 \AA$ is implied by the mean bond length of $1.1164 \pm 0.002 \AA$ and an assumed value for $R_{\mathrm{CH}}\left(1^{0}\right)$ of $1.1134 \pm 0.02 \AA$, the value of $R_{\mathrm{CH}}$ found in a parallel study of hexamethylethane (HME), where all hydrogens are primary. ${ }^{23}$ The experimental value is both in agreement with the calculated value, and significantly larger than the $R_{\mathrm{CH}}\left(2^{\circ} \mathrm{H}\right)$. This grouping of $\mathrm{C}-\mathrm{H}$ bond lengths shows that there is a significant environmental effect on $\mathrm{C}-\mathrm{H}$ bond lengths attributable to geminal interactions. It is notable that the present

${ }^{22}$ R. A. Bonham, L. S. Bartell, and D. A. Kohl, J. Am. Chem. Soc. 81,4765 (1959).

${ }^{23}$ T. L. Boates, thesis, Iowa State University, 1966. 


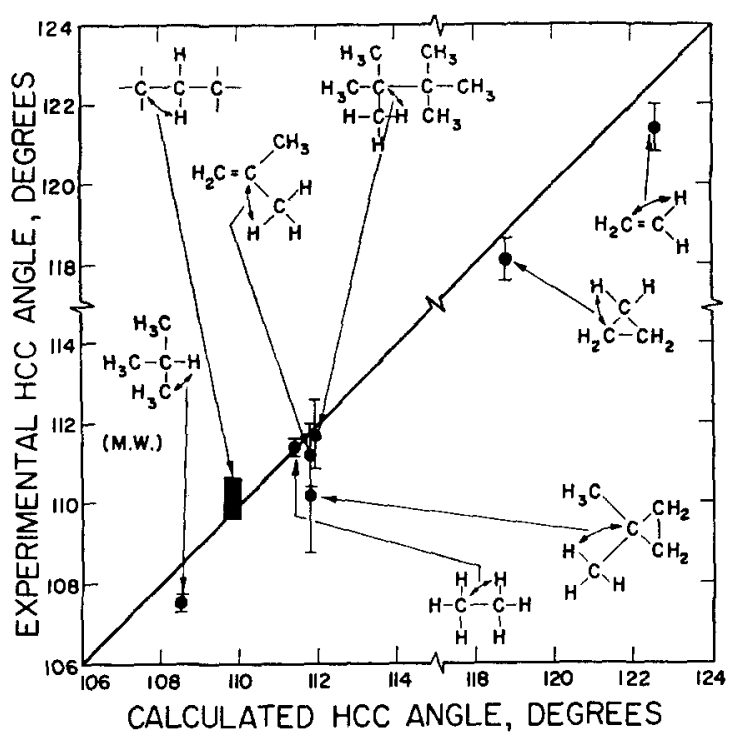

FIG. 3. Comparison of calculated and experimental HCC angles. M.W. denotes a microwave determination. Electron-diffraction angles have been corrected for shrinkage effects. A spread of $\sim 0.5^{\circ}$ was found in the calculated values for HCC angles to secondary hydrogens, as indicated on the graph. Calculated angles are based on tetrahedral $\alpha^{0}$ models except for ethylene in which $\alpha^{0}=120^{\circ}$ was used for angles around the double bond.

correlations were obtained with no assumptions about hybridization, differential hyperconjugation, or any specific electronic factors other than nonbonded interactions. However, the success of the present model does not preclude the importance of the neglected factors, since their approximate influence may be effectively included, under the guise of nonbonded interactions, by hidden compensations built into the nonbonded potential functions chosen.

\section{Bond Angles}

In the case of bond angles, the rough limit of $\pm 2^{\circ}$ on the deviation of calculated angles from corresponding experimental angles represents about $10 \%$ of the total spread of angles observed. This is about the same relative discrepancy observed for the bond lengths. The calculated HCC angles showed the same groupings about characteristic values for primary, secondary, and tertiary hydrogens that the $\mathrm{C}-\mathrm{H}$ bond lengths exhibited. In the same manner that the experimental value for a $\mathrm{C}-\mathrm{H}$ bond to a secondary hydrogen was calculated, a value for the characteristic HCC angle for a secondary hydrogen was derived from the mean $\mathrm{HCC}$ angles reported for the normal alkanes. ${ }^{22}$ The experimental value, $109.7^{\circ} \pm 0.5^{\circ}$ is in good agreement with the calculated $109.8^{\circ}$. It can be seen from Fig. 3 that calculated HCC angles tend to be larger than corresponding experimental values, indicating, perhaps, an imbalance between $\mathrm{C} \cdots \mathrm{H}$ repulsive forces and the force constant associated with an HCC angle. No simple generalization can be drawn from the observed deviations for CCC angles shown in Fig. 4. It should be noted that a common misunderstanding about the decrease in $\mathrm{CCC}$ angle going from the propane to isobutane $e^{24,25}$ is settled by the present calculations. It is sometimes concluded that if the difference between CCC angles in propane and isobutane is steric, the more strained compound isobutane should exhibit the larger CCC angle. A simple resolution of forces demonstrates that the effect, in fact, should be in the opposite direction as observed.

The success of our model in reproducing the structures of simple olefins and cyclopropanes depended somewhat on the reference angles assumed. In going from tetrahedral to $120^{\circ} \alpha^{0}$ 's there were negligible changes in all structural parameters except the angles directly affected, both for the olefins and the cyclopropanes. Table III gives the calculated structures of ethylene and cyclopropane and the pertinent parameters of isobutylene together with experimental structures. In the case of the olefins, there can be no question that the $120^{\circ} \alpha^{0}$ model is more appropriate than the tetrahedral model in terms of agreement with experimental structures. However, the angles exterior to the ring in the cyclopropanes appeared to be much less sensitive to changes in the respective $\alpha^{0}$ 's than were the angles about the double bonds in the olefins, possibly because of the increased number of angles about carbon atoms in the ring compared with those in $\mathrm{C}=\mathrm{C}$ bonds. It is not clear from our results which choice of $\alpha^{0}$ 's is to be preferred for the cyclopropanes. Both choices of $\alpha^{0}$ s did reproduce the shortening observed in $\mathrm{C}-\mathrm{C}$ single bonds adjacent either to $\mathrm{C}=\mathrm{C}$ double bonds or cyclopropane rings, as illustrated by the calculated structures of isobutylene and 1,1-dimethylcyclopropane in Table II and Table III.

\section{Remarks about Deformations}

The predictive ability of the present simple model seems to extend over a large variety of molecules. The

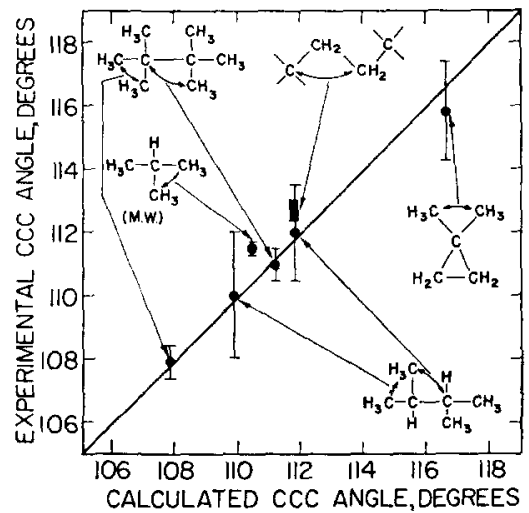

FIG. 4. Comparison of calculated and experimental $\mathrm{CCC}$ angles. $M . W$. denotes a microwave determination. There was a spread in mean CCC angles for $n$-alkanes of $\sim 0.2^{\circ}$, as indicated on the graph. Calculated angles are based on tetrahedral $\alpha^{0}$ models except for isobutylene, in which $\alpha^{0}=120^{\circ}$ was used for angles around the double bond.

24 D. R. Lide, Jr., I. Chem. Phys. 33, 1519 (1960).

${ }^{25}$ D. R. Lide, Jr., J. Chem. Phys. 33, 1514 (1960). 
TABLE III. Calculated structures of ethylene, isobutylene, and cyclopropane for two sets of reference angles.

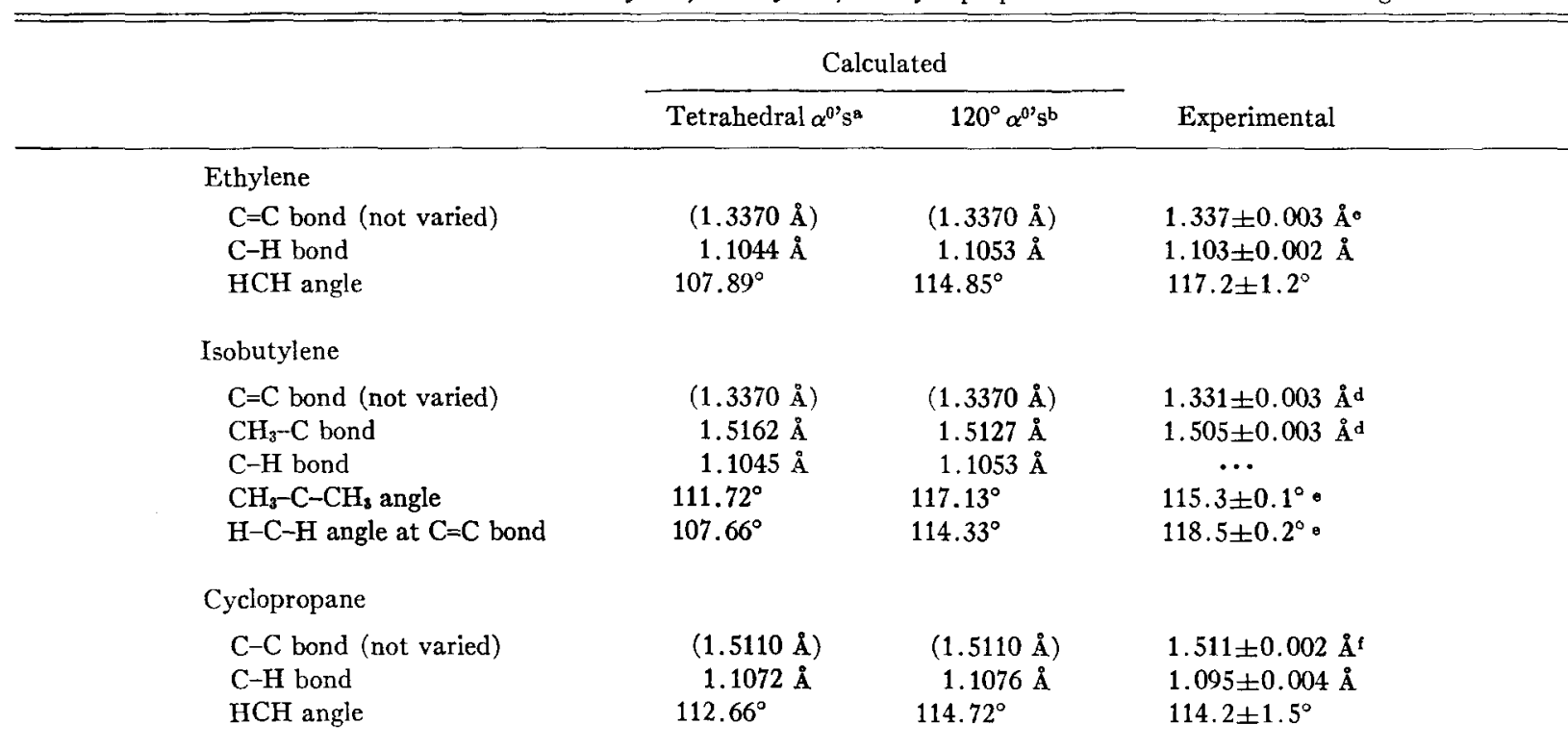

\footnotetext{
${ }^{\mathrm{a}}$ For all three molecules the same force constants, $R^{0} \mathrm{CC}$ and $R^{0} \mathrm{CH}$, as were used for the alkanes were employed. For ethylene and isobutylene the tetrahedral model consists of $\alpha^{0} \mathrm{HCH}=\theta_{t}$, the tetrahedral angle, and $\alpha^{0} \mathrm{CH}_{3}-\mathrm{C}-\mathrm{CH}_{3}=\theta_{t}$, while the remaining angles about the double bond have $\alpha^{0}=1\left(360^{\circ}-\theta_{t}\right)$. For cyclopropane the model consists of $\alpha^{0} \mathrm{HCH}=\theta_{t}$ and the $\alpha^{0} \mathrm{HCC}^{\prime}$ 's equal to the value the $\mathrm{HCC}$ angles have when $\alpha_{\mathrm{CCC}}=60^{\circ}$ and $\alpha_{\mathrm{HCH}}=\theta_{t}$, and $D_{3 h}$ symmetry is assumed for the molecule.

b The $120^{\circ}$ models for the olefins and cyclopropane are analogous to the tetrahedral models with $120^{\circ}$ substituted for $\theta_{i}$ in the definitions in footnote a.
}

model provides detailed predictions of the paths by which individual molecules can find relief from local crowding. The experimental evidence necessary to confirm these predictions is not available in many cases, but where it is available, the agreement is good. On the basis of a representative sample of alkane molecules it is clear that our model predicts deformations from so-called normal parameters which are essentially in agreement with commonly made assumptions about the hierarchy of steric effects. Using the present calculated structures as guides it should be possible to predict at least the directions of deformations in other molecules without performing the entire energy minimization with respect to all structural coordinates and to judge whether observed deformations are of a magnitude which can be accounted for on the basis of nonbonded repulsions alone.

Probably more speculations and calculations have been advanced concerning the deformations at gauche sites in $n$-alkane chains than for any other single kind of steric interaction in hydrocarbon molecules. There is almost universal agreement that the source of the strain is concentrated in a single $\mathrm{H} \cdots \mathrm{H}$ interaction and that the bulk of the relief is achieved by rotations about the three $\mathrm{C}-\mathrm{C}$ bonds between the gauche hydrogens. In isolated gauche configurations, the proper combination of rotations about the three $\mathrm{C}-\mathrm{C}$ bonds effects a fairly large increase in the close $\mathrm{H} \cdot \cdots \mathrm{H}$ dis- c L. S. Bartell and co-workers, Ref. 12 ; gas electron diffraction.

d L. S. Bartell and R. A. Borham, J. Chem, Phys. 32, 824 (1960); gas electron diffraction.

e L. H. Scharpen and V. W. Laurie, J. Chem. Phys. 39, 1732 (1963); microwave, $r_{s}$ structure.

f O. Bastiansen. F. N. Fritsch, and K. Hedberg, Acta Cryst. 17, 538 (1964); gas electron diffraction, reported $r_{a}^{\prime}$ s converted to $r_{a}$ 's.

tance without simultaneously decreasing other repulsive nonbonded distances significantly. Nevertheless, while it may require roughly 10 times as much energy to bend an angle as to make a comparable rotation about a bond, the relative effectiveness of bond bending deformations in relieving steric stress is always enhanced by the greater number of bending degrees of freedom and often by the geometry of the strained site. It is fairly easy to answer the question of whether adjustments in bond angles compete with torsional motions to relieve steric strain for an isolated gauche site. The answer is of some interest since it should represent a lower limit on the contribution of the nontorsional degrees of freedom to the stabilization of strained sites in saturated hydrocarbons. The isolated gauche interaction encounters none of the restrictions on internal rotation found in highly branched alkanes or in rotational isomers of $n$-alkanes with two or more gauche sites adjacent.

Normal butane is the simplest molecule in which a gauche interaction occurs. It takes 19 independent variables to allow each internal coordinate of this molecule to vary under the constraint of $C_{2}$ symmetry. If an energy scale is considered which gives trans- $n$ butane an energy of zero, the energy minimum for gauche- $n$-butane given full freedom is $428 \mathrm{cal} / \mathrm{mole}$, according to the present model. The position of the minimum corresponds to rotations from staggered con- 


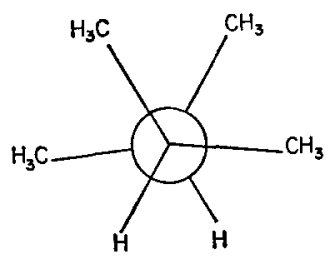

gauche, $C_{2}$

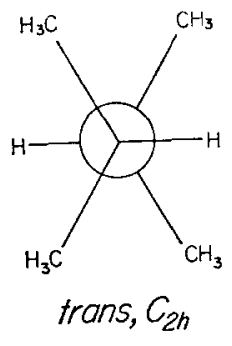

FIG. 5. Newman projections of the rotational isomers of symtetramethylethane.

figurations $\mathrm{s}^{26}$ of $4.4^{\circ}$ about the central $\mathrm{C}-\mathrm{C}$ bond and $-5.0^{\circ}$ about each outer $\mathrm{C}-\mathrm{C}$ bond. If all the internal coordinates of gauche- $n$-butane are fixed at the converged trans- $n$-butane values, and only torsional motion about the three $\mathrm{C}-\mathrm{C}$ bonds is allowed, the energy minimum falls at $5.8^{\circ}$ and $-6.1^{\circ}$ rotations about the central and outer $\mathrm{C}-\mathrm{C}$ bonds, respectively. The energy at the minimum is $551 \mathrm{cal} / \mathrm{mole}$, an increase of 123 $\mathrm{cal} / \mathrm{mole}$ over the model which allowed all internal coordinates to vary. If the molecule is restricted to a single degree of freedom, namely the rotation about the central $\mathrm{C}-\mathrm{C}$ bond, and the remaining internal coordinates are fixed at the values for the trans isomer, the energy minimum falls at a rotation of $10.2^{\circ}$ from staggered, with the energy at the minimum of 723 $\mathrm{cal} / \mathrm{mole}$. With all the reference internal rotations equal to $60^{\circ}$, gauche- $n$-butane has an energy of 1119 $\mathrm{cal} / \mathrm{mole}$. Therefore, for this model, complete relaxation in gauche- $n$-butane stabilizes it by $691 \mathrm{cal} / \mathrm{mole}$. The three rotational degrees of freedom alone provide $568 \mathrm{cal} / \mathrm{mole}$ or $82 \%$ of the energy decrease, while the remaining $18 \%$ can be associated with the inclusion of adjustments in bond angles and bond lengths. The magnitude of the effect of allowing total relaxation of all internal coordinates is, of course, sensitive both to the molecule and to the set of force constants and potential functions. Except in unusual cases, however, small adjustments in many parameters can be expected to be significantly more effective in relieving steric strain than larger adjustments in a few parameters. This should be kept in mind when conformational energy differences are being sought.

In a number of molecules, rotation about $\mathrm{C}-\mathrm{C}$ bonds cannot decrease some gauche interactions without increasing others. In these molecules angular deformations can be large. Where several gauche interactions act across the same $\mathrm{C}-\mathrm{C}$ bond, measurable bond stretches are likely to occur. Recently the electron diffraction structures of two such molecules were determined in this laboratory using recently developed techniques for handling complex molecules. ${ }^{23}$ The first was sym-tetramethylethane. The molecule exists in two distinct rotational conformations, one with two

${ }^{26}$ In this paper, a "staggered" configuration is one for which the dihedral angles selected to measure internal rotations are $60^{\circ}$, i.e., the energy from torsional coordinates is zero. gauche $\mathrm{H} \cdots \mathrm{H}$ interactions (the trans form, $C_{2 h}$ symmetry), and the other with three gauche $\mathrm{H} \cdots \mathrm{H}$ interactions (the gauche form, $C_{2}$ symmetry) as illustrated in Fig. 5. Five different $\mathrm{CCC}$ angles are distributed between the two isomers. Unfortunately, it was impossible to resolve them experimentally with sufficient precision to establish the individual variations conclusively. Distortions of the $\mathrm{CC}$ bonds were better characterized, and mean values of the rotations about the bonds were also obtained. Table IV gives the pertinent structural features of TME which illustrate the distortion of the molecule to accommodate its close $\mathrm{H} \cdot \cdot \mathrm{H}$ distances, together with "normal" values of the parameters. The second molecule studied was hexamethylethane. Only one rotational isomer exists, but the mean structure exhibits $D_{3}$ symmetry, the distortion from $D_{3 d}$ proceeding by rotations about the CC bonds. Again, appreciable bond stretches were observed (and predicted by the model), and angular distortions were appreciable. These parameters are given in Table IV along with "normal" values for comparison. The present model appears to overemphasize the torsional motions and underemphasize bond deformations, at least for these two molecules.

It is usually impossible to determine directly from experiments the full set of independent geometric parameters for molecules of any size and complexity. In all but the most favorable cases, the extraction of deformation parameters from experimental data must rely upon simplifying assumptions about the nature of the deformations to be determined. The model described in this paper can, perhaps, serve as a guide, providing a rational basis for the omission of some types of deformations and the inclusion of others in the treatment of experimental data. An example of a simplified set of deformation parameters is that proposed by Bartell and $\mathrm{Kohl}^{27}$ to characterize local structural differences between trans and gauche sites in the normal alkanes. The set consists of an increment, $\epsilon$, in the $\mathrm{CCC}$ angle per gauche interaction across a particular bond, and two rotations, $\phi$ and $\eta$, about the $\mathrm{C}-\mathrm{C}$ bonds defining the gauche configuration. The parameters are illustrated in Fig. 6. It is possible to arrive at crude values for these deformation parameters by fitting suitable expressions to the experimental structures of $n$-butane, $n$-pentane, $n$-hexane, and

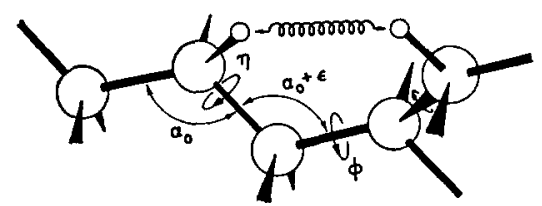

FIG. 6. Deformation parameters for describing gauche conformations in $n$-alkanes. The total gauche dihedral angle is given by $\phi$, while the deviation from trans $\left(180^{\circ}\right.$ dihedral angle) of a bond adjacent to a gauche site is given by $\eta$.

L. S. Bartell and D. A. Kohl, J. Chem. Phys. 39, 3097 (1963). 
TABLE IV. Comparison of calculated and experimental values for selected coordinates of $s y m$-tetramethylethane and hexamethylethane.

\begin{tabular}{|c|c|c|c|c|}
\hline \multirow[b]{2}{*}{ Parameter } & \multicolumn{2}{|c|}{ Calculated } & \multicolumn{2}{|c|}{ Experimental } \\
\hline & Deformed & Normal & Deformed & Normal \\
\hline \multicolumn{5}{|l|}{ Sym-tetramethylethane } \\
\hline Mean CC bond & $1.5468 \AA$ & $1.5438 \AA$ & $1.540 \pm 0.002 \AA$ & $1.533 \pm 0.002 \AA$ \\
\hline $\begin{array}{l}\text { Central CC-terminal CC bond } \\
\text { trans isomer }\end{array}$ & $0.0061 \AA$ & $\cdots$ & $0.005 \pm 0.008 \AA$ & $\cdots$ \\
\hline gauche isomer & $0.0076 \AA$ & $\cdots$ & $0.007 \pm 0.008 \AA$ & $\cdots$ \\
\hline Mean CCC angle & $111.10^{\circ}$ & $110.41^{\circ}$ & $111.4^{\circ} \pm 0.3^{\circ}$ & $111.5^{\circ}$ \\
\hline Mean $\mathrm{CH}_{3}-\mathrm{C}-\mathrm{CH}_{8}$ angle ${ }^{\circ}$ & $109.78^{\circ}$ & $110.41^{\circ}$ & $110^{\circ} \pm 2^{\circ}$ & $111.5^{\circ}$ \\
\hline $\begin{array}{l}\text { Rotation about central CC bond, } \\
\text { gauche isomerd }\end{array}$ & $7.52^{\circ}$ & $\cdots$ & $5^{\circ} \pm 5^{\circ}$ & $\cdots$ \\
\hline \multicolumn{5}{|l|}{ Hexamethylethane } \\
\hline Mean CC bonde & $1.5543 \AA$ & $1.5464 \AA$ & $1.548 \pm 0.002 \AA$ & $1.533 \pm 0.001 \AA$ \\
\hline Central CC-terminal $\mathrm{CC}$ bond & $0.0141 \AA$ & $\cdots$ & $0.041 \pm 0.006 \AA$ & $\cdots$ \\
\hline Angle $\mathrm{CH}_{3}-\mathrm{C}-\mathrm{CH}_{3}{ }^{\mathrm{a}}$ & $107.80^{\circ}$ & $109.47 \cdots{ }^{\circ}$ & $107.92^{\circ} \pm 0.5^{\circ}$ & $109.47 \cdots \circ$ \\
\hline Rotation about central CC bond & $12.6^{\circ}$ (equil.) & $\cdots$ & $5^{\circ} \pm 4^{\circ}$ (mean) & $\cdots$ \\
\hline Rotation about terminal $\mathrm{CC}$ bonds & $5.6^{\circ}$ (equil.) & $\cdots$ & $0^{\circ} \pm 10^{\circ}($ mean $)$ & $\cdots$ \\
\hline
\end{tabular}

In the electron diffraction experiment, the trans isomer was constrained to a $C_{2 h}$ configuration. The calculated structure returned to $C_{2 h}$ from $C_{2}$ distortions.

b The calculated and experimental "normal" values are for isobutane. The experimental bond length was estimated from $r_{0}(0)$ 's for neopentane and the $n$-alkanes.

c The calculated and experimental values for TME refer to averages for the appropriate thermal population of the two isomers. Both "normal"

$n$-heptane. These same parameters can be taken from the calculated structures with results as shown in Table V. The calculated value listed for $\phi$, the rotation about the central $\mathrm{C}-\mathrm{C}$ bond in the gauche configuration, is the average value for a thermal population equimolar in $n$-butane $n$-pentane, $n$-hexane, and $n$-heptane. The value for an isolated gauche configuration, i.e., one either at an end of a molecule or one surrounded by two trans configurations, is $64.5^{\circ}$, indicating a $4.5^{\circ}$ opening of the dihedral angle due to nonbonded interactions. By contrast, for two adjacent gauche configurations in a molecule, the calculated $\phi$ closes down to $59^{\circ}$.

A second example of the sort of simplifying assumptions that could be made in structural analyses of molecules concerns the deviation of methyl groups from threefold symmetry about the $\mathrm{C}-\mathrm{C}$ bond axis. It is possible to treat this deviation as a tilt of the methyl-group axis away from the bond axis, preserving the threefold symmetry of the methyl group itself. Lide has reported that the methyl hydrogens in propane lie in a very nearly equilateral triangle, but the axis of the methyl group apparently does not coincide with the $\mathrm{CC}$ bond axis. ${ }^{25}$ Unfortunately, the uncertainty in the geometry of the $\mathrm{CH}_{3}$ groups was as large as the observed tilt $\left(\sim 1^{\circ}\right)$. The direction of the reported tilt was such as to bring the out-of-plane $\mathrm{CH}_{3}$ hydrogens closer together. On the basis of a steric model a tilt in the opposite direction would be expected. Tilts in the values are for isobutane. The experimental angle $1 \mathrm{~s}$ from the microwave r. structure; D. Lide, Jr., Ref. 24.

d Referred to a configuration in which all three gauche $\mathrm{CCCC}$ dihedral angles are equal.

e Calculated and experimental "normal" values are for neopentane. The experimental bond length is an electron diffraction $r_{0}(0) ; L . S$. Bartell (unpublished data).

f From reference dihedral angles of $60^{\circ}$.

expected direction have been observed in compounds such as $\mathrm{CH}_{3} \mathrm{OH}$ and $\mathrm{CH}_{3} \mathrm{SH}^{28}$ The detailed structures of $\mathrm{CH}_{3}$ groups calculated with the present model suggest a small outward tipping of the $\mathrm{CH}_{3}$ groups. While there is no reason why $\mathrm{CH}_{3}$ groups should preserve threefold symmetry, it might be useful to be able to treat the undoubtedly small deviations from the threefold symmetry about the $\mathrm{C}-\mathrm{C}$ bonds with a single deformation parameter. This and other schemes could readily be tested with the model to determine the validity of the assumptions involved.

\section{B. Strain Energies}

Although the primary purpose of this work was to examine the ability of a mechanical model to predict molecular structures, the formulation of the problem as an energy minimization meant that the total energy of the molecule resulting from steric interactions and resultant strains was calculated as a matter of course in the process of obtaining the desired set of parameters. From these energies it was easy to calculate the influence of nonbonded interactions on rotational and geometrical isomerization energies and rotational barrier heights. It is by no means self-evident that such interactions should be able to account for experimental isomerization energies and trends in barriers but it is

${ }_{28}^{28}$ T. Nishikawa, J. Phys, Soc. Japan 11, 781 (1956) and references therein; T. Kojima and T. Nishikawa, ibid. 12, 680 (1957). 
TABLE V. Deformation parameters for $n$-alkanes.

\begin{tabular}{lcc}
\hline \multicolumn{1}{c}{ Parameter } & Calculated $^{\circ}$ & Experimental $^{\circ}$ \\
\hline CCC angle, mean & $111.73^{\circ}$ & $112.7^{\circ} \pm 0.1_{5}^{\circ}$ \\
$\begin{array}{l}\text { CCC angle, trans configuration, } \\
\alpha_{0}\end{array}$ & $111.02^{\circ}$ & $112.6_{5}^{\circ} \pm 0.3^{\circ}$ \\
$\begin{array}{l}\text { Increment in CCC angle per } \\
\text { gauche interaction, } \epsilon\end{array}$ & $1.23^{\circ}$ & $0.1^{\circ} \pm 1^{\circ}$ \\
$\begin{array}{l}\text { Mean CCCC gauche dihedral } \\
\text { angle, } \phi\end{array}$ & $62.8^{\circ}$ & $61^{\circ} \pm 3^{\circ}$ \\
$\begin{array}{l}\text { Rotation of carbons } 1,4 \text { in } \\
\text { gauche interaction, } \eta\end{array}$ & $4.8^{\circ}$ & $7^{\circ} \pm 4^{\circ}$ \\
\hline
\end{tabular}

See Fig. 6 for further identification of $\alpha_{0}, \epsilon, \phi, \eta$. The notation corresponds to that in Ref. 27.

b All calculated values are for a thermal population of rotational isomers, giving zero weight to isomers with $G G^{\prime}$ sites, and taking an equimolar mixture of $n$-butane, $n$-pentane, $n$-hexane, and $n$-heptane, i.e.. the calculated and experimental parameters are on the same basis.

- Reference 27, values corrected for shrinkage.

informative to make the comparison. This is done below.

- The calculated energies refer to nonvibrating molecules, and contain no temperature dependence, so that they are most suitably compared with thermochemical quantities extrapolated to $0^{\circ} \mathrm{K}$ and corrected for zeropoint vibrational energy. ${ }^{29}$ Corrections of experimental values of $\Delta H_{f}^{\circ}\left(t^{\circ} \mathrm{K}\right)$ to $0^{\circ} \mathrm{K}$ are all based on Pitzer's statistical mechanical calculations of the partition functions for the various hydrocarbon molecules. While errors in the thermodynamic quantities derived from Pitzer's calculations are purported to be small in most instances, it should be kept in mind that a large number of simplifying assumptions, especially about entropy differences between rotational isomers, barrier heights and the effect of internal rotation on other vibrational modes, are embodied in the corrections of the $\Delta H_{f}{ }^{\circ}$ 's to $0^{\circ} \mathrm{K}$. Pitzer estimated ${ }^{30}$ that uncertainties as large as $\pm 2 \mathrm{kcal} / \mathrm{mole}$ were appropriate for the highly branched heptanes and octanes, whereas uncertainties of $< \pm 0.5 \mathrm{kcal} / \mathrm{mole}$ could be assigned to the low molecular weight alkanes. Zero point energies were not available for all the compounds studied. In some cases estimates were made based on the values for related compounds and the observed increment per $\mathrm{CH}_{2}$ group for the alkanes. The uncertainty in the zero point energies introduces an additional source of error for the experimental $\Delta H_{f}^{\circ}\left(0^{\circ} \mathrm{K}\right)$ 's. The errors in isomerization energies derived from differences between $\Delta H_{f}{ }^{\circ}$ 's, are expected to be smaller than the errors in the individual $\Delta H_{f}{ }^{\circ}$ s.

${ }^{29}$ It is a moot point whether corrections for thermal energies and zero-point vibrational energies are profitable in calculations of this sort. See T. L. Allen, J. Chem. Phys. 31, 1039 (1959) and references therein, and K. S. Pitzer and E. Catalano, J. Am. Chem. Soc. 78, 4844 (1956). Our results indicate that the corrections can be significant.

${ }^{30}$ K. S. Pitzer, Chem. Rev. 27, 39 (1940).

\section{Rotational Isomerization Energies}

The $n$-alkanes (and the straight chain segments of branched alkanes) are normally assumed to possess three rotational conformations, gauche, gauche', and trans, about each $\mathrm{C}-\mathrm{C}$ bond. ${ }^{31}$ gauche and gauche' configurations differ only in the direction of their rotation about the $\mathrm{C}-\mathrm{C}$ bond. They may or may not be energetically equivalent, depending upon the rotational configuration of adjacent $\mathrm{C}-\mathrm{C}$ bonds. $\mathrm{A}$ number of calculations have demonstrated that an assortment of models of varying sophistication, but all based on nonbonded interactions, can reproduce the experimental value for the gauche-trans energy difference in $n$-butane. ${ }^{5,8,32}$ Our calculated value for a vibrationless molecule was $428 \mathrm{cal} / \mathrm{mole}$, with the trans form having the lower energy. This can be compared with various experimental determinations. Electron diffraction of gaseous alkanes yields a value for $\Delta G^{\circ}$ of $610 \mathrm{cal} / \mathrm{mole} .^{27}$ Liquid Raman studies of the $n$-alkanes yield the values for $\Delta H$ of $770 \pm 70 \mathrm{cal} /$ mole ( $n$-butane), $450 \pm 60 \mathrm{cal} /$ mole ( $n$-pentane), and $500 \pm 70 \mathrm{cal} /$ mole ( $n$-hexane) ${ }^{33}$ It is of interest to note that the calculated energy differences for the rotational isomers of $n$-pentane indicate that two adjacent gauche configurations actually have slightly less strain energy than two isolated gauche configurations $(2[G T-T T]=990 \mathrm{cal} / \mathrm{mole}$, while $[G G-T T]=794 \mathrm{cal} / \mathrm{mole}$ ). About half of this decrease in strain energy results from the smaller rotational distortions required to relieve the close $\mathrm{H} \cdots \mathrm{H}$ distances. The central CCC angle in $G G n$-pentane is common to both gauche interactions, and consequently is opened to $113.6^{\circ}$, in contrast to the $112.2^{\circ}$ in the $G T$ isomer. This opening of the central $\mathrm{CCC}$ angle is sufficient to increase the close $\mathrm{H} \cdots \mathrm{H}$ distances beyond what the close $\mathrm{H} \cdots \mathrm{H}$ distance is in either

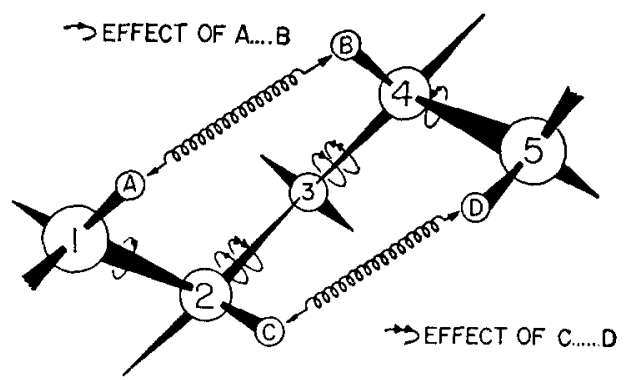

Fic. 7. Directions of rotations for normal relief of gauche interactions in a $G G$ site of a normal alkane. All rotations are for Atom $n$ about the $[n-(n+1)]$ bond. Numbered atoms are carbons, lettered atoms, hydrogens.

${ }^{31}$ Several authors, (Refs. 8 and 9) using models with only torsional degrees of freedom, have reported potential energy minima corresponding to additional stable conformations. Insufficient evidence is available to determine whether they are artifacts of the nonbonded potential functions used and the failure to include bending and stretching degrees of freedom in the potential energy functions being minimized.

${ }^{32}$ See K. S. Pitzer and E.'Catalano, in Ref. 29

${ }^{33}$ G. J. Szasz, N. Sheppard, and D. H. Rank, J. Chem. Phys. 16, $704(1948)$; N. Sheppard and G. J. Szasz, ibid. 17, 86 (1949). 
$G T n$-pentane or in gauche $n$-butane, in spite of the fact that the CCCC dihedral angle in the $G G$ isomer is $59^{\circ}$ and not the $64.5^{\circ}$ found for a single gauche configuration. This difference in rotation about the $\mathrm{CC}$ bonds in going from $G T$ to $G G$ isomers arises in a straightforward manner from the fact that the rotations required about the $2-3$ and 3-4 CC bonds of a five carbon fragment for normal relief of gauche interactions are of opposite directions for the two adjacent gauche interactions. As shown in Fig. 7, the molecule gains little or no relief by rotations about the 2-3 and 3-4 bonds. The stabilization of paired gauche configurations relative to single gauche configurations increases the thermal population of rotational isomers with paired gauche sites. This, in turn, lowers the (gauche-trans) energy difference calculated by averaging over all rotational isomers. It is the latter quantity which is experimentally observable, but both the lowering of the mean $(G-T)$ energy difference and the decrease in the mean gauche dihedral angle predicted by our model are too small to be resolved in present experiments.

A rough value for the free energy of rotational isomerization in TME was recently obtained by electron diffraction. ${ }^{23}$ The trans isomer exhibits two gauche interactions and the gauche isomer, three. Therefore, the isomerization energy is formally equivalent to the energy for converting a trans configuration into a gauche configuration. The experimental distribution of isomers, $60 \%$ gauche and $40 \%$ trans, indicates a $\Delta G^{\circ}$ (gauche-trans) of $170 \mathrm{cal} / \mathrm{mole}$. The electron diffraction $\Delta G^{\circ}$ is consistent with the $\Delta H$ reported for vapor heat capacity and Raman studies of the molecules of less than $200 \mathrm{cal} / \mathrm{mole}$, assuming that, apart from the factor of two favoring the gauche isomer, the entropy difference between isomers is small. ${ }^{34}$ The calculated value of $102 \mathrm{cal} / \mathrm{mole}$ is also compatible with the experimental result. The fact that this pseudo gauchetrans energy difference is much smaller than other values for gauche-trans energies is not surprising when one considers the geometries of the two isomers. First, in both isomers the projections of the $\mathrm{CH}_{3}-\mathrm{C}-\mathrm{CH}_{3}$ angles on a plane perpendicular to the central $\mathrm{C}-\mathrm{C}$ bond exceed $120^{\circ}$. This means that without any rotation about the central bond the mean gauche dihedral angle in the gauche isomer is greater than $60^{\circ}$ while for the trans isomer it is less than $60^{\circ}$. Furthermore, the gauche isomer can relieve two of its gauche interactions at the expense of the third by a rotation about the central $\mathrm{C}-\mathrm{C}$ bond. In contrast, the trans isomer can relieve neither of its gauche interactions by rotation about the central $\mathrm{C}-\mathrm{C}$ bond, and $C_{2 h}$ remains the symmetry of the most stable form. The net effect is a destabilization of gauche sites in the trans isomer relative

${ }^{34}$ G. J. Szasz and N. Sheppard, J. Chem. Phys. 17, 93 (1949); D. W. Scott, J. P. McCullough, K. D. Williamson, and G. Waddington, J. Am. Chem. Soc. 73, 1707 (1951); J. K. Brown and N. Sheppard, J. Chem. Phys. 19, 976 (1951). to those in the gauche isomer. Calculated structures of both isomers included methyl rotations of magnitudes comparable to those found at gauche sites in other molecules. While it is not easy to assess by inspection the effect of methyl rotations on the rotational isomerization energy of TME, it is probable that the effect is small. Whatever the origin of the low isomerization energy in TME, it is clear that the strain energy to be associated with gauche configurations is not constant, but is strongly dependent on the environment of the particular configuration. Calculations which ignore this fact may lead to misleading results.

\section{Geometrical Isomerization Energies}

While it is possible to ascribe the major part of the energy difference between rotational isomers to a single nonbonded $\mathrm{H} \cdots \mathrm{H}$ interaction, the energy differences between geometrical isomers are much more complicated functions of the interactions involved. As was pointed out several years ago, ${ }^{10}$ the fact that isomerization energies are small (on the order of a few kilocalories) should not be taken as evidence that intramolecular nonbonded repulsions are small. These isomerization energies can arise from small differences between large quantities. If the potential functions used for the present calculations are able to give reasonable values for isomerization energies, extensive cancellations of fairly large repulsive terms must occur. The leading nonbonded terms in the isomerization energy for converting a $n$-alkane into its corresponding iso form are

$$
V_{\text {norma1 }}-V_{\text {iso }}=2 V_{\mathrm{CH}}-V_{\mathrm{HH}}-V_{\mathrm{CC}},
$$

where the $V_{n b}$ 's are evaluated at appropriate geminal nonbonded distances. This expression merely reflects changes in the numbers of the various geminal interactions in going from a normal to an iso configuration. Allen's expression for the $\mathrm{C}-\mathrm{C}$ interaction energy in alkanes is just the negative of this expression. ${ }^{4}$ of the 14 sets of potential functions with which Allen evaluated this quantity, only three gave even the right sign; one of these was the set used in the present calculations. It must be said that this agreement was not entirely accidental since the construction of the functions (8) - (10) above was biased by considerations of correlating isomerization energies. Still, the quantity of Eq. (13) is only a rough guide to the calculated isomerization energy when all interactions in the molecules are considered, and when the geometrical parameters are allowed to readjust upon isomerization. Table VI shows calculated $\Delta E_{\mathrm{isom}}$ 's compared with experimental $\Delta H\left(0^{\circ} \mathrm{K}\right)$ 's, corrected for zero-point energies. In all the cases calculated, the lower energy form was correctly predicted, and the magnitude of the energy difference was at least reasonable. No cases of small experimental isomerization energy $(<0.5 \mathrm{kcal} / \mathrm{mole})$ were examined with the model because small experi- 
TABLE VI. Isomerization and zero point energies of saturated hydrocarbons."

\begin{tabular}{|c|c|c|c|c|}
\hline & \multirow{2}{*}{$\begin{array}{l}\text { Abbreviation } \\
\text { for Fig. } 8\end{array}$} & \multicolumn{2}{|c|}{$\begin{array}{c}\text { Isomerization energy, } \\
\mathrm{kcal} / \mathrm{mole}^{\circ}\end{array}$} & \multirow{2}{*}{$\begin{array}{l}\text { Zero poin } \\
\text { energy, } \\
\mathrm{kcal} / \mathrm{mole}\end{array}$} \\
\hline & & Calculated & Experimentald & \\
\hline$n$-Butane & -4 & $0.0 \ldots$ & $0.0 \ldots$ & $80.62 t$ \\
\hline Isobutane & $2 m 3$ & 1.336 & 1.39 & $80.38^{t}$ \\
\hline$n$-Pentane & -5 & $0.0 \ldots$ & $0.0 \ldots$ & $98.32 t$ \\
\hline Isopentane & $2 m 4$ & $0.82^{\circ}$ & 0.87 & $97.61^{f}$ \\
\hline Neopentane & $22 m 3$ & 3.966 & 3.05 & $97.30^{t}$ \\
\hline$n$-Hexane & -6 & $0.0 \ldots$ & $0.0 \cdots$ & $116.02 x$ \\
\hline Isohexane & $2 m 5$ & $0.68 \mathrm{e}$ & 0.46 & $115.31^{\mathrm{h}}$ \\
\hline 2,3-Dimethylbutane (TME) & $23 m 4$ & 1.002 & $(1.0)$ & $(115.2)^{i}$ \\
\hline 2,2-Dimethylbutane & $22 m 4$ & 2.645 & 2.91 & $115.19^{\mathrm{j}}$ \\
\hline$n$-Heptane & -7 & $0.0 \ldots$ & $0.0 \cdots$ & $133.72^{\alpha}$ \\
\hline Isoheptane & $2 m 6$ & $0.72^{\circ}$ & 0.51 & $133.01^{\mathrm{b}}$ \\
\hline 2,2-Dimethylpentane & $22 m 5$ & 2.491 & 2.62 & $132.89 \mathrm{k}$ \\
\hline 2,2,3-Trimethylbutane & $223 m 4$ & 2.12 & $(1.8)$ & $(132.4)^{1}$ \\
\hline$n$-Octane & -8 & $0.0 \ldots$ & $0.0 \cdots$ & $151.42 *$ \\
\hline Iso-octane & $2 m 7$ & $0.71^{\bullet}$ & 0.51 & $150.71^{\mathrm{h}}$ \\
\hline 2,2-Dimethylhexane & $22 m 6$ & 2.61 & 2.20 & $150.59^{k}$ \\
\hline $2,2,3,3$-Tetramethylbutane (HME) & $2233 m 4$ & 1.950 & (1.19) & $(149.57)^{\mathbf{m}}$ \\
\hline
\end{tabular}

a The experimental heats of formation and the calculated energies used to obtain the isomerization energies were those for the rotational isomers of lowest energy. Isomerization energies are for (normal -isomer). Parentheses surround values with unusually large uncertainties.

$b$ The notation is that of Allen, who adapted it from Platt. See Ref. 5.

- The experimental isomerization energies listed have already been corrected for zero-point energy differences.

${ }^{d}$ Heats of formation at $0^{\circ} \mathrm{K}$ were taken from F. D. Rossini, K. S. Pitzer, R. L. Arnett, R. M. Braun, and G. C. Pimentel, Selected Values of Physical and Thermodynamic Properties of Hydrocarbons and Related Compounds (Carnegie Press, Pittsburgh, Pa., 1953), $w$ tables.

The calculations for the isoalkanes were done on the rotational isomers with $C_{\text {\& }}$ symmetry. An energy difference of $500 \mathrm{cal} /$ mole between the $C_{\text {s }}$ Isomer and the lowest energy $C_{1}$ isomer was assumed in each case. The

mental isomerization energies were only reported between pairs of branched alkanes for which the uncertainties in the individual $\Delta H_{f}^{\circ}\left(0^{\circ} \mathrm{K}\right)$ 's were large. In these cases comparisons of calculated isomerization energies with the corresponding experimental values would not be a fair test of the predictive ability of the model.

In calculations of this sort, the emphasis on the results is more properly placed on trends and patterns in the data than on the actual calculated values. For this reason, an effort was made to see first whether any recognizable patterns existed in the experimental heats of formation, and if so, whether the calculated results reproduced these patterns. Graphs were made of both the calculated potential energies and the experimental heats of formation (at $0^{\circ} \mathrm{K}$, corrected for zero-point energies) as functions of the number of carbon atoms in the molecule. Since the increment in energy per $\mathrm{CH}_{2}$ unit is considerably larger than isomerization energies for the alkanes, a plot of unmodified energies would tend to obscure any trends in isomerization energies, i.e., differences between individual energies of geometrical isomers. In order to make the graphs more sensitive to fine details, the experimental heats of formation and energy of the $C_{1}$ isomer of isopentane was calculated as a check and found to be $470 \mathrm{cal} / \mathrm{mole}$ lower than the $C$ * form.

I See Ref. 32.

8 Calculated from zero-point energy (ZPE) ( $n$-pentane) on the basis of the increment per $\mathrm{CH}_{2}$ unit of $17.7 \mathrm{kcal} / \mathrm{mole}$ given in Ref. 32 .

h ZPE (isopentane) $+(n-5) \times 17.7 \mathrm{kcal} / \mathrm{mole} ; n=$ number of carbon atoms. See Ref. 32 .

I $\mathrm{ZPE}(2,3$-dimethylbutane $) \approx Z \mathrm{PE} \quad(2,2$-Dimethylbutane). See footnote $j$.

j T. L. Allen, J. Chem. Phys. 31, 1039 (1959).

k ZPE $(2,2$-dimethylbutane $)+(n-6) \times 17.7 \mathrm{kcal} /$ mole. See Ref. 32 .

1 ZPE (2,2-dimethylpentane) $-0.5 \mathrm{kcal}$.

m $Z P E(2,2$-dimethylhexane) - [ZPE ( $n$-pentane)-ZPE (neopentane)].

the calculated energies were levelled by the following process. An energy increment, $\delta$, per carbon atom was calculated by taking one-sixth the energy difference between $n$-octane and ethane. A correction $(n-2) \delta$ to the energy of an alkane with $n$ carbon atoms was made to each molecule to be represented on the graphs. Leveling corrections for calculated energies differed from those of the experimental energies, of course, because intrinsic bond energies play no role in the present theoretical model. Results are shown in Fig. 8. The deviation of methane from the level of the $n$-alkanes can be rationalized in a fairly simple manner. In going from methane to ethane by addition of a $\mathrm{CH}_{2}$ group, there is an increase of six geminal nonbonded interactions, all of which are $\mathrm{C} \cdots \mathrm{H}$ interactions. The addition of a $\mathrm{CH}_{2}$ group to any higher $n$-alkane brings an increase of one $\mathrm{C} \cdots \mathrm{C}$, one $\mathrm{H} \cdots \mathrm{H}$, and four $\mathrm{C} \cdots \mathrm{H}$ geminal nonbonded interactions. The difference between these two sets of interactions associated with the addition of a $\mathrm{CH}_{2}$ group to methane or an $n$-alkane is $\left(2 V_{\mathrm{CH}}-V_{\mathrm{CC}}-V_{\mathrm{HH}}\right)$. This can be recognized as the same expression for estimating the isomerization energy for chain branching in alkanes. Consequently, the energy difference between methane and ethane is com- 
parable to the energy difference between iso $-\mathrm{C}_{n} \mathrm{H}_{2 n+2}$ and $n-\mathrm{C}_{n+1} \mathrm{H}_{2 n+4}$. Since the $n$-alkanes exhibited a regular increase in energy with increasing chain length it seemed reasonable to suppose that various series involving branching at a particular site with repeated additions of $\mathrm{CH}_{2}$ groups to the straight chain segment should also show regular trends in energies. The 2-methyland the 2,2-dimethyl-series on the graphs indicate some regularity. With the exception of neopentane, the calculated values appear to follow the pattern exhibited by the experimental heats of formation. The inclusion of corrections for zero point energies, rough though the corrections were, improved the correlation significantly.

\section{Rotational Barriers}

The origin of barriers to internal rotation in the hydrocarbons has not yet been interpreted in a completely rigorous fashion, but recent calculations ${ }^{35}$ indicate that slightly shielded proton-proton repulsions can account for the entire barrier in ethane and related molecules. While the working assumptions embodied in our model are not entirely compatible with this result, it is possible that the model can nonetheless correlate the observed variations in barrier heights in the paraffin hydrocarbons, and thus provide a useful measure of the relative ease of rotation in various steric environments.

Since calculations based on our model involved only nonvibrating structures, comparison of calculated barriers with experimental values, in which the observable is related to torsional modes of vibration, should be made with caution. The calculated barriers are arrived at by minimizing the energy with respect to all internal coordinates except the rotation angle under consideration for fixed values of that rotation angle. In propane and neopentane, where the motion of the dependent methyl(s) is coupled to that of the independent methyl by nonbonded interactions, the relative motions of the methyl groups in our model should be analogous to the methyl rotations associated with the torsional mode of lowest energy for the molecules.

The contributions of nonbonded interactions and of relaxation of internal coordinates to the barriers in propane, $n$-butane, neopentane, and hexamethylethane were investigated. Energies of "relaxed" and "unrelaxed" configurations are reported. "Unrelaxed" configurations are those for which the internal coordinates (except for the rotation angle) were kept constant at the values found for the minimum energy configuration.

For ethane, the energy difference ("relaxed") between the eclipsed and staggered forms was about $270 \mathrm{cal} / \mathrm{mole}$. (The energy difference referred to does not include the $2730 \mathrm{cal} / \mathrm{mole}$ intrinsic barrier, of course.) The calculated energies, for the rotation angle

${ }^{35}$ J. P. Lowe and R. G Parr, J. Chem. Phys. 44, 3001 (1961).

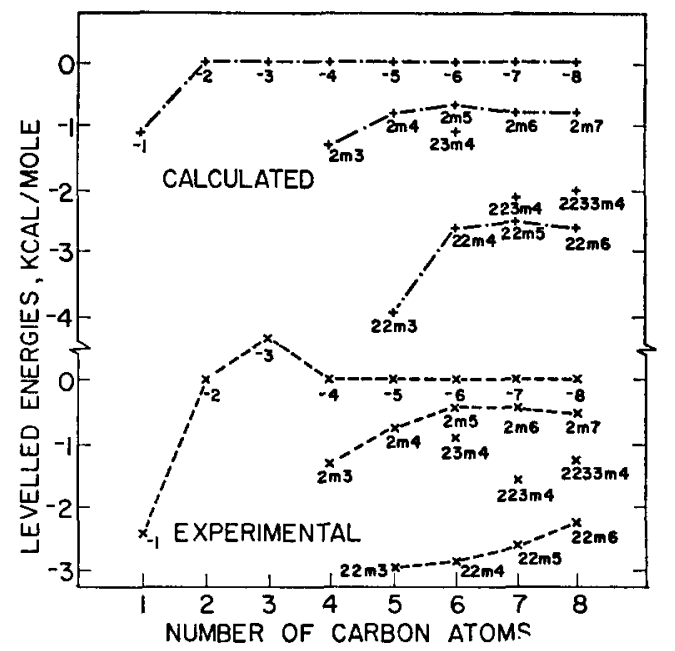

Fig. 8. Comparison of calculated and experimental leveled energies of saturated hydrocarbons. The energies plotted are measured from ethane, which is taken to have zero energy. In each case the energy is that of lowest energy rotational isomer of the given molecule. Experimental energies have been corrected for zero-point vibrational energies. See text for description of leveling process and Table VI for notation used to identify individual molecules represented.

taken in $10^{\circ}$ increments, were fitted with a function of the form

$$
V=\frac{1}{2} V_{3}^{\prime}(1-\cos 3 \theta)+\frac{1}{2} V_{6}(1-\cos 6 \theta)
$$

with $V_{3}{ }^{\prime}=274.4 \mathrm{cal} / \mathrm{mole}$ and $V_{6}=-2.5 \mathrm{cal} / \mathrm{mole}$. The "unrelaxed" energy difference (eclipsed-staggered) was less than $5 \mathrm{cal} / \mathrm{mole}$ higher than the "relaxed" contribution to the barrier even though the change in bonded energy with rotation angle contributed nothing to the "unrelaxed" energies but more than $50 \%$ of the calculated energy difference of the relaxed model.

In propane the effect of allowing relaxation of the internal coordinates was slightly greater than in ethane. The "environmental" contribution to the energy difference between eclipsed and staggered forms was 614 $\mathrm{cal} / \mathrm{mole}$ if no relaxation was allowed, and $572 \mathrm{cal} / \mathrm{mole}$ with relaxation. The "relaxed" value corresponds to a total barrier in propane of $3302 \mathrm{cal} / \mathrm{mole}$. Experimental measures of the barrier range from $3100-3600$ $\mathrm{cal} / \mathrm{mole}$, with $3100-3300 \mathrm{cal} / \mathrm{mole}$ more probable than the higher values. ${ }^{36} \mathrm{~A}$ fit of the calculated energies for propane ("relaxed") to Eq. (14) yielded the values $V_{3}{ }^{\prime}=571.8 \mathrm{cal} / \mathrm{mole}$ and $V_{6}=2.6 \mathrm{cal} / \mathrm{mole}$. In the calculations, one "independent" methyl was rotated in uniform increments while the other "dependent" methyl was allowed to adjust its conformation until an energy minimum was achieved. The methyl-methyl interaction was slight, however, and the "dependent" methyl group rotated from its perfectly staggered

${ }^{36}$ G. B. Kistiakowsky, J. R. Lacher, and W. W. Ransom, J. Chem. Phys. 6, 900 (1938); J. D. Kemp and C. J. Egan, J. Ám. Chem. Soc. 60,1521 (1938); G. B. Kistiakowsky and W. W. Rice, J. Chem. Phys. 8, 610 (1940). 


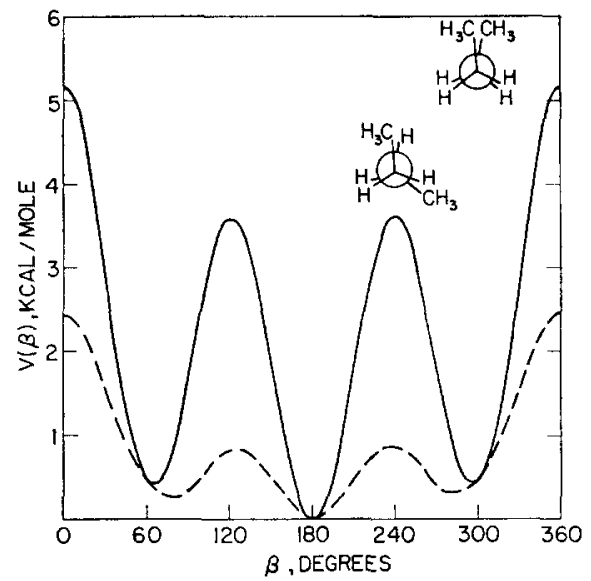

Fic. 9. Calculated barrier to rotation about the central $\mathrm{CC}$ bond in $n$-butane. The solid curve represents the total energy of the molecule as given by Eq. (2) in the text. The dashed curve represents the contribution of nonbonded interactions and relaxation of all internal coordinates except $\beta$, i.e., everything except the $2700 \mathrm{cal}$ "intrinsic" barrier assumed for the central bond.

reference configuration by a maximum of $1^{\circ}$. This rotation contributes a very small, positive, sixfold term to the barrier.

The same type of behavior that was found in propane also was observed in neopentane, but it was exaggerated by the presence of two additional methyl groups which further hindered rotation. The "environmental" part of the barrier was fitted with a $V_{3}{ }^{\prime}=1368 \mathrm{cal} / \mathrm{mole}$ and $V_{6}=12.9 \mathrm{cal} / \mathrm{mole}$. This corresponds to a total barrier of $4098 \mathrm{cal} / \mathrm{mole}$. The experimental barrier is $4300 \mathrm{cal} / \mathrm{mole}^{37}$ Coupling between rotations of the methyl groups again introduces a small sixfold term into the barrier. The "dependent" methyl groups rotate a maximum of $1.2^{\circ}$ from staggered configurations.

As is well known the barrier to rotation about the central $\mathrm{C}-\mathrm{C}$ bond in $n$-butane is not threefold symmetric, and there are two distinct configurations of minimum energy, the trans and the gauche forms. Perforce there are two distinct eclipsed forms (representing energy maxima), also. In one form, methyl groups eclipse hydrogen atoms; in the other, methyl groups eclipse each other. The most natural explanation for the destabilization of the gauche configuration is that steric interactions between the methyl groups in the gauche isomer are greater than the interactions between two methyl groups and the hydrogen atoms opposite them in the trans isomer. On this basis it is to be expected that the eclipsing of one methyl group by another will require more energy than the eclipsing of two methyl groups by hydrogen atoms. Unfortunately, there is no direct experimental confirmation of this conclusion. Pitzer's educated guess of $3600 \mathrm{cal} / \mathrm{mole}$ and $4400 \mathrm{cal} / \mathrm{mole}$ for the energies of the eclipsed forms

${ }^{37}$ K. S. Pitzer and J. E. Kilpatrick, Chem. Rev. 39, 435 (1946). of $n$-butane ${ }^{30}$ was sufficiently good to allow corrections for the effects of hindered rotation on thermodynamic functions to be made. However, thermodynamic properties of the molecule are relatively insensitive to the barrier height assumed, and even more insensitive to a small energy difference between two eclipsed forms. A comparison of the barrier calculated using the present model with the barrier assumed by Pitzer for his statistical mechanical calculations must be made with strong reservations about the significance of any agreement or disagreement between the two.

The barrier for $n$-butane was calculated both for the "relaxed" and "unrelaxed" models. In this case, the close approach of two methyl groups in the course of rotation about the 2-3 bond was relieved significantly by the relaxation of the remaining internal coordinates. The calculated "environmental" energy differences (eclipsed-staggered, trans) for the "relaxed" model were $848 \mathrm{cal} / \mathrm{mole}$ for the methyl groups eclipsing hydrogen atoms and $2432 \mathrm{cal} / \mathrm{mole}$ for methyl groups eclipsing each other. These values, when added to the $2730 \mathrm{cal} / \mathrm{mole}$ "intrinsic" part of the barrier, yield energy maxima of $3578 \mathrm{cal} / \mathrm{mole}$ and $5162 \mathrm{cal} / \mathrm{mole}$ (see Fig. 9). The energy differences for the "unrelaxed" model were $939 \mathrm{cal} / \mathrm{mole}$ and $5536 \mathrm{cal} / \mathrm{mole}$ (given in the same order as for the "relaxed" model above). These values correspond to energy maxima of 3666 and $8266 \mathrm{cal} / \mathrm{mole}$, respectively.

A calculation of the potential energy function for rotation about the central C-C bond in "relaxed" hexamethylethane was performed to see whether the severe crowding of hydrogens, which was sufficient to lower the symmetry from $D_{3 d}$ to $D_{3}$ for the minimum energy configuration, also gave a high barrier. The potential function possesses six minima corresponding to equal energy and lying at $\pm 12.6^{\circ}$ (calculated) from the staggered configurations. The energy difference between the equilibrium $D_{3}$ configurations and the perfectly staggered $D_{3 d}$ configurations was only 316 $\mathrm{cal} / \mathrm{mole}$. In spite of the fact that the gauche interactions in this molecule are considerably more repulsive than in the isolated gauche conformation in $n$-butane, a circumstance which destabilizes the staggered conformations of HME, the total barrier (eclipsed-equilibrium) was quite high $(8400 \mathrm{cal} / \mathrm{mole}$ including the $2730-\mathrm{cal} / \mathrm{mole}$ "intrinsic" contribution). Thermodynamic measurements of the barrier in this molecule ${ }^{38}$ assumed a $D_{3 d}$ configuration. The data were sufficient only to yield an average barrier height, assuming seven equivalent (energetically) rotations and no interactions between rotating groups. The value obtained was $4700 \mathrm{cal} /$ mole. However, in the light of the present evidence, the model of Scott et al. represents too great an oversimplification of the potential energy function of molecules to yield the desired barrier.

${ }^{38}$ D. W. Scott, D. R. Douslin, M. E. Gross, G. D. Oliver, and H. M. Huffman, J. Am. Chem. Soc. 74, 883 (1952). 


\section{CONCLUDING REMARKS}

This research demonstrates that it is possible to reproduce trends in molecular structures, isomerization energies, and rotational barriers for a variety of hydrocarbons using a model force field in which the only sources of variations are the nonbonded interactions. There is no reason to believe, however, that the nonbonded potential functions adopted represent more than an extremely crude approximation to an optimum choice. A limited study was made with several alternative combinations of quadratic constants and nonbonded functions as discussed below. In each case, the results were in poorer agreement with experiment than were the results reported in the foregoing text based on the initial choice of force field.

In one study, calculations were made on the alkanes through butane using the original quadratic force constants, but substituting another set of nonbonded functions. The new functions were of the (exp-6) form, and were obtained by a semiempirical method developed by Williams ${ }^{39}$ to deduce intermolecular interactions. Although the Williams functions worked very well in representing intermolecular properties, and although the original functions and the Williams functions were of the same general magnitude and hardness, the Williams functions gave poor structural parameters for molecules and the wrong sign for the isomerization energy of isobutane. Apparently, in intramolecular problems, the balance between nonbonded functions is more critical than their actual magnitudes.

A second study involved an attempt to modify the quadratic constants for bond angles and bond lengths in order to correct the diagonal $F$ matrix elements for the fact that the spectroscopic U-B nonbonded constants $(F$ 's) were somewhat smaller than the constants implied by the nonbonded functions at the appropriate internuclear distances. The modified quadratic constants, when used with the original nonbonded functions and reference angles gave a very poor fit to the structure of ethane, so the treatment was not pursued further.

Comparison of calculated structures of methane and ethane with experimental data provides a simple but fairly sensitive test on the basis of which some com-

\footnotetext{
${ }^{39}$ D. E. Williams, J. Chem. Phys. 45, 3770 (1966). Our calculations were based on a preliminary set of functions calculated by Williams in the course of his development of the technique. They are in kilocalories per mole: $V_{\mathrm{CC}}=38340 \exp (-3.546 r)-350 r-6$; $V_{\mathrm{CH}}=8149 \exp (-3.54 r)-129.6 r^{-8} ; V_{\mathrm{HA}}=1732 \exp (-3.535 r)-$ $48.0 r \rightarrow$.
}

binations of force constants and nonbonded functions can be rejected. An additional simple test of the nonbonded functions is the application of Eq. (13), which affords a rough estimate of the energetic balance among the functions. Application of Eq. (13) to the original functions yields an approximate isomerization energy of $+1 \mathrm{kcal} / \mathrm{mole}$ for the iso-alkanes, as compared with the experimental range of $0.5-1.4 \mathrm{kcal} / \mathrm{mole}$. The Williams functions predict a value of $-1 \mathrm{kcal} / \mathrm{mole}$. The functions used by Scott and Scheraga ${ }^{8}$ to investigate rotational conformations in $n$-alkanes imply a value of $-10 \mathrm{kcal} / \mathrm{mole}$ for the isomerization energy, and on this basis alone can be regarded as unsuitable for the model. An extremely strong $\mathrm{H} \cdot \mathrm{HH}$ function is the source of the imbalance in this case. McCullough and McMahon, in their study of rotational states in $n$-hexane, used functions which are undesirable for the same reason. ${ }^{9,40}$

In summary, whether the discrepancies between experimental and our calculated values simply reflect an imperfect choice of force constants and nonbonded potential functions, or whether they signify the neglect of more specific interactions is a question which cannot be answered from available data. In view of the neglect of popularly accepted specific interactions we should, perhaps, be more surprised at the successes of the model than at its failures.

A number of applications of the model remain to be investigated. It would be especially worthwhile to examine some of the branched hydrocarbons which have $G G^{\prime}$ sites, and therefore must accommodate extremely repulsive $\mathrm{H} \cdots \mathrm{H}$ interactions. Some of these molecules which have high symmetries or otherwise favorable geometries could be studied by electron diffraction as a test of the model's predictive powers.

\section{ACKNOWLEDGMENTS}

This work was initiated and carried out in part in the Ames Laboratory of the U.S. Atomic Energy Commission, Iowa State University. We gratefully acknowledge this source of support. We also wish to thank the Horace H. Rackham School of Graduate Studies of the University of Michigan for a Faculty Research Grant which expedited the concluding phases of this research.

${ }^{40}$ McCullough and McMahon used only $\mathrm{H} \cdots \mathrm{H}$ and $\mathrm{C} \cdots \mathrm{H}$ interactions in their treatment. A C $\cdots \mathrm{C}$ function to complete their set can be generated by using a simple geometric mean rule for the repulsive and attractive terms. 